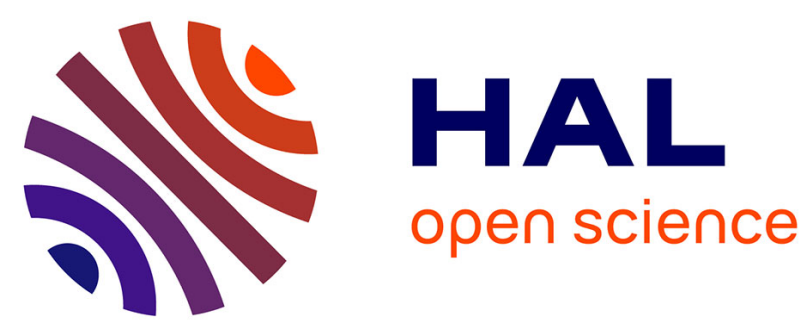

\title{
Targeted energy transfer between a Rotor and a Morse oscillator: A model for selective chemical dissociation
}

Antony Memboeuf, Serge Aubry

\section{To cite this version:}

Antony Memboeuf, Serge Aubry. Targeted energy transfer between a Rotor and a Morse oscillator: A model for selective chemical dissociation. Physica D: Nonlinear Phenomena, 2005, 207 (1-2), pp.1-23. 10.1016/j.physd.2005.05.016 . hal-00713109

\section{HAL Id: hal-00713109 \\ https://hal.univ-brest.fr/hal-00713109}

Submitted on 29 Jun 2012

HAL is a multi-disciplinary open access archive for the deposit and dissemination of scientific research documents, whether they are published or not. The documents may come from teaching and research institutions in France or abroad, or from public or private research centers.
L'archive ouverte pluridisciplinaire HAL, est destinée au dépôt et à la diffusion de documents scientifiques de niveau recherche, publiés ou non, émanant des établissements d'enseignement et de recherche français ou étrangers, des laboratoires publics ou privés. 


\title{
Targeted Energy Transfer \\ between a Rotor and a Morse oscillator: \\ A model for selective chemical dissociation
}

\author{
A. Memboeuf ${ }^{\mathrm{a}}$ and S. Aubry ${ }^{\mathrm{b}}$ \\ ${ }^{a}$ Centre for Research in Molecular Modelisation, Parc Initialis \\ Avenue Copernic-Bât. Materia Nova 7000 Mons, Belgium \\ ${ }^{\mathrm{b}}$ Laboratoire Léon Brillouin (CEA-CNRS), CEA Saclay \\ 91191 Gif-sur-Yvette Cedex, France
}

Standard Kramers theory of chemical reactions involve a coupling with a Langevin thermal bath which intrinsically forbids the possible existence of Discrete Breathers (i.e. local modes). However, it is now known that in complex systems, that energy may focus for long time as Discrete Breathers (local mode). In very special systems, Targeted Energy Transfer may occur subsequently to another selected site and induce an ultraselective chemical reaction operating at low temperature. The dynamics of the reaction is non brownian but highly coherent along a specific path in the phase space where the system is nearly integrable (chemical expressway). A simple toy model illustrating this idea is reduced to a rotor weakly coupled to a Morse oscillator (supposed to represent two specific local modes in a complex system) which are appropriately tuned for Targeted Energy Transfer. When the nonlinearities of the two oscillators are appropriately tuned one with each other, and when the rotor is initially rotating with a frequency resonant with those of the Morse oscillator at rest, the energy of the rotor is almost completely transferred to the Morse oscillator and induces chemical dissociation. The periodic oscillations of the rotor and Morse oscillator remain coherent and their frequencies simultaneously vary, but always remain resonant.

This process is analytically described within an integrable approximation. Numerical investigations of this model confirm that in the appropriate conditions, the particle in the Morse oscillator is indeed promptly ejected at infinity with a finite velocity (chemical dissociation) despite some chaotic transient manifesting imperfect integrability. 


\section{Introduction}

In his famous pioneering work $[1,2]$ published in 1940, Hendrik Kramers understood ordinary chemical reactions, as the climbing of an energy barrier between two energy wells in a large configuration space where the first well represents the state of the reactant molecules and the second well those of the product molecules. In standard theories, chemical reactions are still essentially governed by a thermal (Brownian) motion in a potential. This space is defined by the reaction coordinates which describe the microscopic configuration of the reacting system. Many detailed investigations have been performed during the last decades for specific chemical reactions where energy landscape, basin and saddle point in energy (transition states) were accurately calculated.

For ordinary chemical reactions which are assisted by a thermal Brownian force, the system remains trapped over long times in basins around deep local minima of the potential energy surface and there are rare but essential events where the system succeeds to reach and to pass saddle points (transition states) between two different basins of the potential energy surface. When the energy landscape becomes complex, the chemical reaction follows a random pathway which connects different basins. These chemical reactions are non selective and their products are determined according to statistical rules.

However, it is well-known that many chemical reactions in biosystems are induced by enzymes which do not obey Arrhenius laws ( as it should be as a consequence of Kramers theory). Many open problems concern DNA transcription through bubble opening, protein folding and biological machines which involve bond breaking/formation with a high degree of selectivity and specificity in conformational changes. Biosystems easily operate through specific enzymes, specific chemical reactions at room temperature and pressure condition which otherwise would not be feasible elseway or be feasible only under extreme temperature and pressure conditions. Let mention as an example among many others, the well-studied nitrogenase enzyme ([3]) which synthetises ammonia from atmospheric nitrogen in certain microorganisms while industrial ammonia synthesis is still a quite expansive process.

Many biochemical reactions are fueled by the hydrolysis of ATP which requires this prerequisite reaction energy be not waisted in the thermal bath but kept in a coherent form for boosting selectively subsequent reactions. Despite an abundant literature mostly experimental, theoretical explanations for the physical mechanism used by these enzymes based on conventional schemes but are not really convincing. Enzymes should be viewed as complex dynamical systems, especially built by living cells in which nonrandom but coherent processes play an essential role at some crucial steps for selecting specific chemical reactions. It is thus challenging to suggest new possible non-Kramers mechanisms for 
chemical reactions based on specific properties of special dynamical systems.

A flaw of the standard Kramers theory is that it assumes instantaneous dissipation that is the frequency spectrum of the Brownian force generated by the Langevin thermal bath is uniform (white) without any gaps and cut-off. As a result, the reaction energies which are released at the intermediate stages of the reaction process, should be quickly dissipate into heat in the thermal bath. The evolution of the system is Markovian, with independent probability rates at each reaction step. Although these assumptions turn out to be sufficient (and convenient) for describing most ordinary chemical reactions, the possibility for the system to exhibit coherent processes requiring long term memory is systematically killed out in Kramers theory.

It has been recognized as a ubiquitous phenomena that in many nonlinear systems, energy may remain focused and trapped for long times as excited local modes (Discrete Breathers or DBs) which are coherent time periodic oscillations. These conditions specifically require that the thermal bath is not uniform in frequency and thus cannot be an ideal Langevin thermal bath as considered in the Kramers theory. Frequency gaps (resp. discreteness) in the phonon spectrum are required for the existence DBs (or Intraband DBs (IDBs) see later). Phenomena of energy focusing may trigger subsequently selected chemical reactions (occurring within the life time of these Discrete Breathers) which would be highly unprobable within the original Kramers theory.

The purpose of the present paper is to show from the theoretical point of view that such coherent mechanisms could exist. Such processes could operate at low temperature, non-Kramers ultraselective chemical reaction along special pathways in te phase space we call chemical expressways (see fig.1). These chemical expressways are viewed as thin regions in the complete phase space (described with both the configuration coordinates and their associate momenta) where the dynamics of the system remains locally highly coherent that is nearly integrable. Although their existence requires exceptional welltuned conditions, we believe that they could precisely be chosen by biosystems because of their high selectivity and efficiency.

We already proposed a model for Ultrafast Electron Transfer where a quantum electron is coupled to a classical thermal bath [4] and which exhibits such a chemical expressway. This theory uses variations of the same basic concept of Targeted Energy Transfer as we shall use here but modified for Targeted Electron Transfer considering the quantum wavepacket of the electron instead of a classical local mode. In that paper, we return in the context of the Kramers model which was originally classical. This approach is valid when the quantum electronic state always remain in adiabatic equilibrium with respect to the atomic configuration and then determine the potential energy of this atomic configuration without being explicitly involved in the atomic dynamics. 


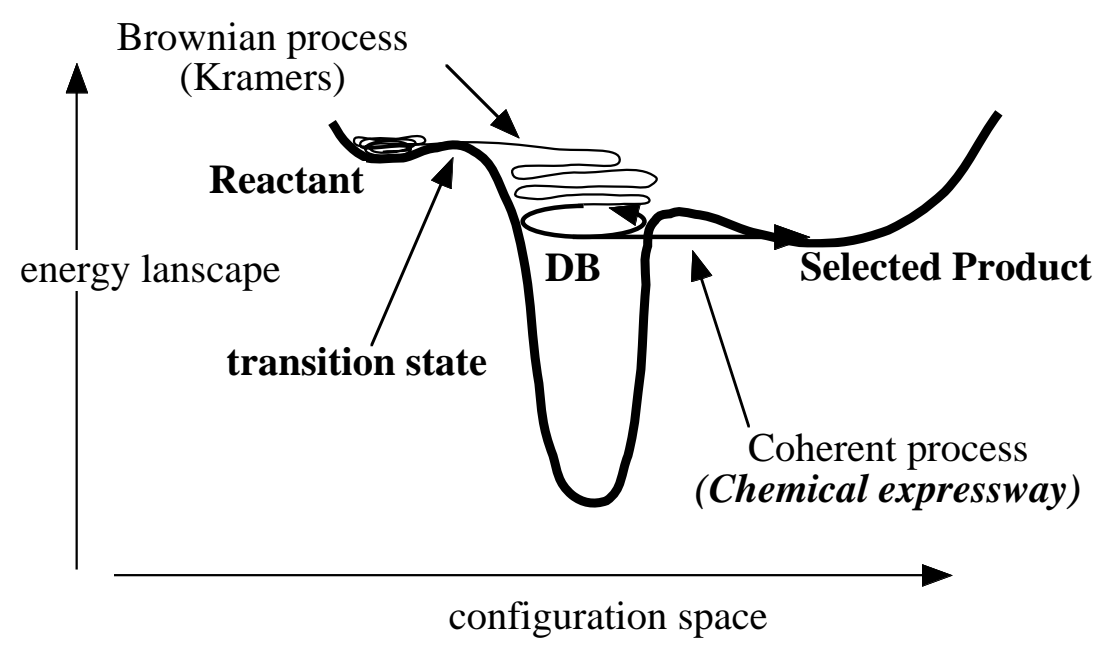

Fig. 1. Scheme of a chemical expressway. A prerequisite Kramers reaction has released some energy which spontaneously focus as a local mode (DB). In well-built systems, this focused energy may be transferred selectively to another selected local mode (targeted energy transfer) where it triggers a subsequent reaction to a selected product. chemical expressway.

This paper presents a simple mechanical toy model which exhibits a chemical expressway. It simply consists of a rotor and a Morse oscillator which are weakly coupled. For a specific choice of the rotor inertia momentum and of its initial energy, the rotor energy is spontaneously and coherently transferred to the chemical bond and induces at zero temperature a very fast chemical reaction (dissociation) requiring in principle the focusing of a large energy.

It is thus useful in this context to make first a preliminary review on the most recent developments on energy localization and DBs in complex systems which justifies that nonlinear oscillators as involved in our theory could represent local modes in complex systems. Thus, the next section 2 is devoted to a brief review of the recent achievements on energy focusing as local modes. Section 3 , discusses possible mechanisms for the selective transport of focused energy through a complex system (targeted energy transfer). Section 4 describes the general theory of Targeted Energy Transfer between two nonlinear oscillators (without damping). Section 5 applies this new concept to the simple RotorMorse oscillator system. Section 6 describes the numerical observation of the ultrafast chemical dissociation at zero temperature and thus yields an example of chemical expressways. Section 7 discusses the effect of thermal and quantum fluctuations not included in the model and its relevance for real system and section 8 summarizes the paper. 


\section{Energy Localization in Complex Systems: Discrete Breathers ( Local Modes)}

Actually, numerous studies show that finite polyatomic molecules seen as a set of non-linear coupled oscillators is not an ergodic system, but instead, they form a mixed phase space with regular and chaotic regions [5,6]. A particularly important and ubiquitous phenomena in large complex systems, is the occurrence of spontaneous energy localization which can be associated with the excitation of local modes. Transitions from normal modes (extended motions) to local modes were observed spectroscopically in molecules but their existence was already claimed in chemistry in the twenties [7] although ignored during several decades as mentioned in [8].

These local modes exist in finite size nonlinear systems as well as in infinite discrete and nonlinear lattices which is even more striking. Beside the usual chemist terminology local mode, they were independently called Intrinsic Localized Mode $(I L M)$ or Discrete Breathers $(D B)$ in physics. There existence was claimed first on the basis of numerical simulations [9] and confirmed subsequently in many kinds of large discrete nonlinear systems. The numerical experiment is very simple. If a big packet of energy is injected locally that is on a single site or on few neighboring sites (for example as kinetic energy) in a nonlinear discrete model at zero (or low) temperature which may sustain such solutions, it is found that only a small part of this energy spread out by phonon radiation and after a relatively short chaotic transient that most the energy remains localized. The initial wave packet spontaneously relax as a time periodic local mode (Discrete Breather or DB) [10-12]. If the amplitude of this localized oscillations is well above the thermal noise, it could persist out of thermal equilibrium over quite long time before complete relaxation.

They were considered as approximate solutions till they have been rigorously proven to be exact solutions in various kinds of nonlinear models [13-17]. Their existence proof requires both the discreteness and the nonlinearity of the system but does not require necessarily its spatial periodicity. It was initially required that the frequency of these DBs and its harmonics should belong to gaps of the linear phonon spectrum because otherwise it would radiate away energy by the phonons so that the DB would rapidly decay. However, this argument holds only for systems with a continuous linear spectrum where the phonons are extended.

This is not the case in infinite non periodic systems with discrete phonon spectrum where there is Anderson localization for the linear phonons. However, it is still often believed that because of the resonances are dense, any local mode with a frequency in the phonon spectrum, should decay very fast. Nevertheless, we have conjectured on the basis of detailed numerical analysis of DB 
bifurcations on finite systems with increasing size that families of Intraband Discrete Breathers (IDB) at a given spatial location may persist when their frequencies belong to a fat Cantor set imbedded inside the discrete phonon spectrum $[18,19]$.

We give some arguments here. A family of IDBs is the family of nonlinear modes associated with a given linear Anderson (normal) mode when its amplitude becomes finite. When the frequency of the IDB becomes almost resonant with another normal mode, this IDB is destroyed (through a bifurcation) as an exact localized solution in a certain interval of frequency around its frequency ${ }^{1}$. Since the normal modes frequencies are supposed to be dense, the frequency intervals where a given IDB disappears are dense. However, the width of the gap associated with each resonance decays exponentially as a function of the spatial distance between this resonant normal mode and the IDB. The consequence is that the total sum of the gap widths where the IDB disappears is convergent and finite and moreover it is smaller than the measure of the whole phonon spectrum. As a result the IDB of the considered family persists in the complementary set of this dense set of gaps which is a fat (i.e. nonvanishing measure) Cantor set of frequencies. Moreover, the measure density on this Cantor set tends to full measure at the small amplitude limit where the IDB tends to be the linear Anderson mode at the corresponding location ${ }^{2}$. As a result, there is a Cantor set domain of frequency for each IDB family attached to each Anderson mode. This Cantor set depends on the spatial location of the IDB family which changes its relative distance to the resonances. Unlike standard extraband DBs which form continuous families as a function of their amplitude (or frequency), each IDBs family is defined on a discontinuous set with many frequency gaps although most of them are negligible.

This general conjecture is supported by a rigorous proof done early by Albanèse and Fröhlich [20] on a particular nonlinear and random model. They proved that each Anderson mode is the zero amplitude limit of a family of localized time periodic solutions (IDB) with nonvanishing amplitude and a frequency belonging to a fat Cantor set. However, they did not prove the destruction the IDBs in the frequency gaps associated with each resonance.

Moreover, in a paper in preparation [22] coauthored by one of us, we shall analyze the existence of IDBs in models of anharmonic oscillators coupled to

1 Extended time periodic solutions also appear [18] but with an infinite (extensive) energy and thus have to be discarded in the present context.

2 There is a strong similarity of structure between this fat Cantor set and the frequency domain of existence of KAM tori for example in the standard map. In the later case, the resonance gaps are determined by the dense set of rational numbers instead of an a priori arbitrary dense set of normal modes frequencies. Close to integrability, the fat Cantor set domain of existence of KAM tori also goes to full measure. 
a harmonic phonon bath with dense spectrum. It will be proven rigorously that when the frequency spectrum of this bath is continuous, there are no exact IDB solutions with frequencies in the phonon spectrum. Thus, if the continuous spectrum ranges uniformly from frequency zero to infinity IDBs cannot exist at any frequency. This result confirms that IDBs intrinsically cannot exist in models coupled with a Langevin thermal bath (which have a continuous, gapless and unbounded spectrum). On contrary, when the spectrum of the phonon bath is discrete, IDBs may exist with a frequency inside the phonon spectrum provided it belongs to a fat Cantor set imbedded in the dense phonon spectrum with the structure described above. It will be also conjectured on the base of strong arguments that these IDBs are linearly stable but in a fat sub-Cantor set only which proves their physical relevance for applications.

In summary, despite direct experimental evidence are still lacking, we already obtained quite strong theoretical arguments that local modes (DBs or IDBs) may persist as classical exact solutions in complex realistic models. It also demonstrates that the standard modeling of a complex environment by a dissipative Langevin phonon bath may become physically incorrect in complex random systems if the phonon spectrum is discrete. DBs and IDBs should manifest in the dynamical properties of the system providing the temperature is not too high or equivalently when they sustain locally amounts of energy which are much larger than the expected average thermal energy.

Many of these exact DBs solutions (local modes) are linearly stable which mean they do not interact with the small fluctuations treated as linear. However, they do interact at higher order. The consequence is that at finite temperature, DBs exchange energy with the thermal fluctuations and decay but provided the temperature is not too large, the life-time of this local modes may remain unexpectedly long. Although the explicit analytic calculation of these life-time remains an open problem, many numerical evidences show that very generally infinite or large complex systems (spatially periodic or not) may spontaneously localize large amount of energy (substantially larger than the thermal energy) as time periodic coherent states (DB, IDB or local mode). These excited states persist over long time much longer than those expected from standard phonon relaxation.

It is now essential to note that chemical reactions ( or photoexcitations....) release their energy locally (for example from the hydrolysis of ATP)[21]. Since this energy is generally substantially larger than the ambient thermal energy, these conditions seem to be quite favorable for exciting long living and large amplitude local modes (provided they exist). as mentioned above. It would be quite inefficient for the biosystems that this energy be immediately spread out and thermalized in the environment but better to keep a substantial part of this energy focused for triggering specific chemical reactions. Otherwise they would be quite unprobable at the average ambient temperature. Local modes 
(IDB) are surely an efficient way for coherent energy trapping and focusing as vibrational energy.

It should be noted that the idea that energy focusing is necessary for understanding chemical reactions in living systems, is not new although it now gained strong support because of the recent developments confirming the ubiquity of this phenomena. Long ago Davydov already proposed in the 70's a very interesting model for energy localization and transportation in $\alpha$ helix of protein which anticipates on some respects, the new theory of local modes [23,24]. The Davydov model also assumes that energy could be stored as an amide I quantum vibrational exciton coupled to low frequency phonons (Davydov soliton). Indeed, experimental evidence of these excitations has been recently obtained in crystalline acetanilide [25,26] and later in $\alpha$-helix [27]. Energy transportation is obtained in the Davydov model by assuming these solitons are moving freely along the $\alpha$ helix . However, local modes or DB are generally not mobile except in very peculiar conditions. It requires the Davydov soliton to be rather spatially extended which is not confirmed by experiments. It also requires the $\alpha$ helix is very regular with spatial periodicity which are conditions difficult to find in biosystems. Moreover, Davydov soliton mobility is reversible and not selective.

DBs models were also considered some years ago by Peyrard and Bishop [28] for understanding the DNA denaturation as a function of temperature. Another model was proposed later [29] for the problem of chemical dissociation induced by DBs but none of them were considering coherent processes for DB transfer as we propose here.

\section{Selective and Coherent Transfer of Discrete Breathers}

After discussing that spontaneous energy focusing should be ubiquitous in complex nonlinear systems, let now assume that some prerequisite chemical reaction ( or another type of excitation e.g. photoexcitation etc...) has focused a substantial amount of vibrational energy into a specific local mode. If this energy is not involved immediately in a fast coherent process for a next step chemical reaction, this energy will relax after some time as thermal energy and lost for assisting the subsequent steps of the chemical reaction (which means in the scheme of fig.1 that the system relaxes to the lowest minimum).

However, specially well-built systems (biosystems?) may take advantage of this focused energy for generating subsequently and selectively a fast coherent process (chemical expressway) which brings the system into a state which would be very unlikely reached within a brownian (Kramers) process. This specific chemical reaction may be induced by a fast and selective energy transfer of 
the focused energy from the initial local mode to another specific location (another local mode).

The only possible mechanism for a fast propagation of vibrational (or rotational) energy which is efficient even at small coupling involves resonances. The simplest and well-known example consists of two weakly coupled resonant harmonic oscillators. Any amount of energy injected on one of them is completely transferred to the other one after a certain time proportional to the coupling (and because of time reversibility the energy subsequently oscillates back and forth). However, in that case no extra feature which could be interesting, occurs because the system is linear.

Actually this energy transfer generally breaks down for two weakly coupled anharmonic oscillators (or two local modes). Indeed, for nonlinear oscillators, the condition of initial resonance is not sufficient for complete transfer. The reason is that because of the anharmonicity, the frequencies of these oscillators change as a function of the transferred energy. Generally, they do not remain equal which breaks the resonance and consequently stops the energy transfer. However, if the varying frequencies of the two nonlinear oscillators remain equal during the whole transfer, resonance persists and the energy transfer is complete as in the linear case. This condition is fulfilled when beside the condition of resonance at the initial time, another condition is required on the nonlinear terms [30] which is described in the next.

Then, when a selected amount of energy is injected on the first one, this energy is completely transferred to the second one after some time while the frequencies of the two nonlinear oscillators both vary but persistently remain equal. Note the important difference with the linear case where energy transfer occurs for any amount.

Two anharmonic oscillators are said to be conjugated when they are appropriately tuned one with each other for complete transfer of a certain amount of energy. We called this phenomena Targeted Energy Transfer (TET) [30].

Thus, conjugacy is an extension to nonlinear oscillators of the well-known concept of linear resonance but it is quite more selective. It is more exceptional for two nonlinear oscillators (or local modes) chosen at random to be conjugate at a given energy than simply linearly resonant. This rare situation is nevertheless highly interesting because it is precisely the reason which could produce a high selectivity in complex systems with many modes. Many other modes could be possibly linearly resonant but only the conjugate one could capture most the energy. It produces a dynamical lock-and-key phenomena where a given local mode could recognize in a large environment a unique acceptor for transferring most its vibrational energy.

Thus, finding a conjugate local mode in the environment of a given local mode 
would a priori a rare event in a purely random system but on contrary could be frequent in biosystems (enzymes) because of its selectivity. Because of that, many reactions in biochemistry should be understood very differently from those of standard chemistry. Some billion years of evolution and mutations have selected and improved the most efficient enzymes for specific reaction pathways producing the specific biomolecules (among many possible others) needed by the living cells.

However, let us note that as explained in the previous section, a given local mode (IDB) in a complex system belongs to a family of exact solutions which is generally discontinuous in frequency. If we assume that there are no normal mode with frequency in the interval of variation of its frequency, this family is continuous and this normal mode family can be represented by the family of solutions of an effective single nonlinear oscillator.

There could also exist many normal modes which become linearly resonant in the interval of frequency variation. But as we said, linear resonance is not sufficient for substantial energy transfer considering that generally the local modes families associated with these normal modes are not conjugate with the excited anharmonic local mode. Nevertheless, there are small energy losses when passing such resonances. They could be viewed as a kind of frequency dependent damping in the system dynamics. In any case, as soon the temperature is nonvanishing, there are also higher order interactions of the local mode with the normal modes which also damp this effective nonlinear oscillator.

At the present stage, we have written down the TET condition only in the undamped case. An effective damping could be empirically included in the effective nonlinear oscillators representing two local modes for taking into account the nonconjugate modes and the thermal fluctuations. Then, the conjugacy conditions between two damped nonlinear oscillators should be modified for taking into account the energy losses during energy transfer, but we have not yet analytically written down this correction. Nevertheless, we already performed numerical tests [31] of TET between conjugate local modes in a large random system without any correction and found that could TET persist in the presence of a small damping (obtained by an extra coupling with a resonant harmonic phonon band) providing it is not too large. Moreover, TET could become irreversible for an appropriate tuning of the energy dissipation during transfer. It is thus simpler and more pedagogical at this stage to describe TET without damping before considering more complex situations. 


\section{Analytic Conditions for Targeted Energy Transfer}

For understanding the conditions for TET, we consider the ideal case without any damping and thermal random force in a reduced model which consists of two weakly coupled nonlinear oscillators called donor (D) and acceptor (A) respectively, representing two specific local modes in a complex system. It is convenient to describe the Hamiltonian $\mathcal{H}$ of our reduced system with their standard action-angle variables $\left(I_{D}, \theta_{D}\right)$ and $\left(I_{A}, \theta_{A}\right)$ respectively

$$
\mathcal{H}=H_{D}\left(I_{D}\right)+H_{A}\left(I_{A}\right)+\lambda W\left(I_{D}, I_{A}, \theta_{D}, \theta_{A}\right)
$$

$H_{D}\left(I_{D}\right)$ (resp. $\left.H_{A}\left(I_{A}\right)\right)$ is the energy of the uncoupled local mode (D) (res.(A)) as a function of its action $I_{D}\left(\operatorname{resp} . I_{A}\right)^{3}$. The coupling potential $\lambda W\left(I_{D}, I_{A}, \theta_{D}, \theta_{A}\right)$ is arbitrary but small and thus depends for convenience on the small parameter $\lambda$.

Let us note that the problem of energy transfer between two anharmonic oscillators was investigated earlier but in the general regime without and where $\lambda$ is not small $[32,33]$. The system was chaotic and the process of energy transfer was found stochastic. We consider here the nearly integrable situation where $\lambda$ is small.

At $\lambda=0$, this Hamiltonian (1) with $2+2$ degree of freedom is integrable since both $I_{D}$ and $I_{A}$ are time invariant. As soon as $\lambda \neq 0$, the system becomes generally non integrable and exhibits chaotic trajectories. However, at small $\lambda$, the chaotic manifestations are negligible over relatively long time scale compared to the time for TET (if any). The ideal TET condition which will be given in the following, means that at the uncoupled limit $\lambda=0$, there is a particular energy $E_{T}$ such when $E_{T}=H_{D}\left(I_{D}\right)+H_{A}\left(I_{A}\right)$, the frequencies of the Donor and the Acceptor oscillator $H_{D}^{\prime}\left(I_{D}\right)=H_{A}^{\prime}\left(I_{A}\right)$ remains equal, which means that we have a continuum of degenerate tori.

In that region of the phase space, Hamiltonian (1) can be accurately approximate by another integrable nonlinear system but where the two nonlinear oscillators remain coupled.

Considering $\lambda$ small, $I_{D}$ and $I_{A}$ are slow variables since their time derivatives are proportional to $\lambda$. If in addition, we consider the region in the phase space where the system is close to resonance, that is $\omega_{D}=H_{D}^{\prime}\left(I_{D}\right) \approx \omega_{A}=H_{A}^{\prime}\left(I_{A}\right)$, the angle difference $\theta_{D}-\theta_{A}$ becomes also a slow variable. It is then convenient

\footnotetext{
3 In the case this family of local modes (IDBs) would be discontinuous as mentioned in the second section, we should consider $H_{D}\left(I_{D}\right)$ as the envelope of the energy interpolating the gaps considering that damping terms should be also introduced in the dynamics as also suggested above.
} 
to perform a canonical change of variables where the new pairs of conjugate variables are $\left(\theta=\theta_{D}-\theta_{A}, I=\left(I_{D}-I_{A}\right) / 2\right.$, and $\left(\theta_{0}=\theta_{D}+\theta_{A}, I_{0}=\left(I_{D}+I_{A}\right) / 2\right)$.

The only fast variable in the Hamiltonian (1) is $\theta_{0}$ while the three other variables $I_{0}, I, \theta$ are slow. In that regime, the adiabatic approximation is valid. The dynamics of the system can be well described by the Hamiltonian $\mathcal{H}_{a}$ obtained by averaging the initial Hamiltonian $\mathcal{H}$ over the fast variable $\theta_{0}$ while the slow variables $I_{0}, I, \theta$ are supposed to be fixed. This averaged Hamiltonian is

$$
\mathcal{H}_{a}=H_{D}\left(I_{0}+I\right)+H_{A}\left(I_{0}-I\right)+\lambda C\left(I_{0}, I, \theta\right)
$$

where $C\left(I_{0}, I, \theta\right)=\left\langle W\left(I_{0}+I, I_{0}-I,\left(\theta_{0}+\theta\right) / 2,\left(\theta_{0}-\theta\right) / 2\right)\right\rangle_{\theta_{0}}$ is the average on $\theta_{0}$.

This Hamiltonian $\mathcal{H}_{a}$ with $2+2$ degrees of freedom is independent of $\theta_{0}$. Consequently, the total action $2 I_{0}=I_{D}+I_{A}$ is a time invariant (in addition to the total energy $\mathcal{H}_{a}$ ) and Hamiltonian $\mathcal{H}_{a}$ is integrable.

Since the total action is conserved, the energy of the system during the transfer is $H_{D}\left(I_{0}+I\right)+H_{A}\left(I_{0}-I\right)$ where $I$ is the difference of action between donor and acceptor. Since the coupling energy is small, it should be almost constant. Let us assume first that for a certain value of $I_{0}$, the detuning function

$$
\epsilon\left(I_{0}, I\right)=H_{D}\left(2 I_{0}\right)-H_{D}\left(I_{0}+I\right)-H_{A}\left(I_{0}-I\right)
$$

is zero for all $I$. This condition implies that $H_{D}^{\prime}\left(I_{0}+I\right)=H_{A}^{\prime}\left(I_{0}-I\right)$. It is equivalent to say the frequencies of the donor and acceptor oscillators, remains equal at constant action ( or at constant energy). In that situation, the dynamics of the system is driven only by the perturbative term which is coupling function

$$
\begin{aligned}
& \dot{I}=-\lambda \frac{\partial C\left(I_{0}, I, \theta\right)}{\partial \theta} \\
& \dot{\theta}=\lambda \frac{\partial C\left(I_{0}, I, \theta\right)}{\partial I}
\end{aligned}
$$

It has been shown [30] that when $C\left(I_{0}, I, \theta\right)$, as a function of $\theta$, is well-behaved, that is sinelike (i.e. with one maximum and one minimum only per period), the solution $I(t)$ with initial condition $I(0)=I_{0}$ (all the energy on the donor) varies periodically between $I_{0}$ and $-I_{0}$ (all the energy on the acceptor).

Actually, the phase space restricted to $(I, \theta)$ has the topology of a sphere where $I=\sin \alpha$ determines the latitude $\alpha$ and $\theta$ is the longitude of a point of the sphere. $\theta$ is undefined at the two poles at $I=I_{0}$ and $I=-I_{0}$. A trajectory which connects the two poles is a TET solution.

When the energies on the donor and on the acceptor at constant action are 
equal $H_{D}\left(2 I_{0}\right)=H_{A}\left(2 I_{0}\right)$, complete energy transfer is still possible when $\epsilon\left(I_{0}, I\right)$ is not strictly zero but small. The trajectory which connects the two poles is obtained by writing the energy conservation $H_{D}\left(I_{0}+I\right)-H_{A}\left(I_{0}-\right.$ $I)+\lambda C\left(I_{0}, I, \theta\right)=H_{D}\left(2 I_{0}\right)$. If it is connected in one piece, the TET solution exists. The necessary and sufficient condition for that is

$$
\min _{\theta} \lambda C\left(I_{0}, I, \theta\right)<\epsilon\left(I_{0}, I\right)<\max _{\theta} \lambda C\left(I_{0}, I, \theta\right)
$$

This condition is fulfilled when the coupling $|\lambda|$ is larger than a small nonzero value. When, it is not fulfilled, the line becomes disconnected which means that an intermediate energy barrier appears and blocks the complete energy transfer. This transition is first order.

We note that for fulfilling the TET condition (6), a smaller coupling requires a smaller detuning function. This condition is more compelling for the anharmonicity of the nonlinear oscillators but the selectivity in initial energy or action becomes sharper as the coupling becomes smaller.

The obtained trajectory which yields TET is generally time periodic for the averaged Hamiltonian (2) at fixed $I_{0}$. It rotates around the sphere along the line connecting the two poles of the reduced spherical phase space $(I, \theta)$ with the frequency $\omega_{T E T}$ proportional to the coupling parameter $\lambda$.

The corresponding trajectory for the initial Hamiltonian (1) also depends on the variable $\theta_{0}$ which oscillates at the fast frequency $\omega_{0}=\partial \mathcal{H}_{a} / \partial I_{0}$. This frequency depends on $I_{0}, I, \theta$ and thus varies periodically at the slow frequency $\omega_{T E T}$. In any case, the corresponding trajectory in the original phase space $\left(I_{D}, \theta_{D}, I_{A}, \theta_{A}\right)$ is generally quasiperiodic on a smooth torus (unless accidentally it closes on a single loop). If the difference between Hamiltonian (1) and its approximation (2) is small, in this region of the phase space, many approximate torus of the TET trajectory may persist as a KAM torus for the original nonintegrable Hamiltonian . In that case, the adiabatic approximation remains qualitatively correct even at long time.

In case, the corresponding KAM torus is broken up because of frequency resonance, it mixes with a thin stochastic layer where the real TET trajectory integrated with Hamiltonian (1) are chaotic at long time scale.

\section{Targeted Energy Transfer in the Rotor-Morse Oscillator System}

We consider now an example of system where TET may occur. The simple Morse oscillator [34] describing chemical bonds for diatomic covalent molecules could also model the local mode associated with bond vibrations. The Morse 
potential as a function of the bond length $R$, has the form [35]

$$
V(R)=D_{e}\left(1-e^{-a\left(R-R_{e}\right)}\right)^{2} \approx \frac{1}{2} m_{e} \omega_{e}^{2}\left(R-R_{e}\right)^{2}+\ldots
$$

where $D_{e}$ is the dissociation energy, $R_{e}$ the equilibrium distance of the bond and $a=\sqrt{\frac{m_{e}}{2 D_{e}}} \omega_{e}$ where $m_{e}$ is the reduced mass of the bounded atoms, and $\omega_{e}$, the harmonic frequency of the bond vibration at small amplitude.

Rotor are also quite common in biomolecules. They correspond to torsional modes. Rotor and Morse oscillator turn out to be perfectly conjugate oscillators at the dissociation energy of the Morse oscillator when the inertia momentum of the rotor has a well defined value. According to the above theory of TET, we have to represent first these oscillators in action-angle variables.

The Hamiltonian of a Morse oscillator can be written

$$
H_{M}=\frac{p^{2}}{2}+\frac{1}{2}\left(e^{-u}-1\right)^{2}
$$

where we choose as units of mass $m_{e}$, of distance $1 / a$ and of energy $2 D_{e}$ ( then $\omega_{e}=1$ in order to get a Hamiltonian without parameters)

This classical oscillator (8) has explicit bounded time periodic solutions which are

$$
u(t)=\ln \frac{1-\sin \beta \cos \omega_{M} t}{\cos ^{2} \beta}
$$

where $0 \leq \beta<\pi / 2$ is a arbitrary parameter. The energy (8) of this solution is $H_{M}=\frac{1}{2} \sin ^{2} \beta$ and its frequency $\omega_{M}=\cos \beta$. Since $\omega_{M}=d H_{M} / d I_{M}=$ $\sqrt{1-2 H_{M}}$, it readily comes out that this Hamiltonian becomes

$$
H_{M}=I_{M}-\frac{1}{2} I_{M}^{2}
$$

in action-angle variables $I_{M}, \theta_{M}$ where $0 \leq I_{M}=1-\cos \beta<1$

It is also straightforward to obtain explicitly $u\left(I_{M}, \theta_{M}\right)$ as a function of the action angle variables from eq.9.

$$
u\left(I_{M}, \theta_{M}\right)=\ln \frac{1-\sin \beta\left(I_{M}\right) \cos \theta_{M}}{\cos ^{2} \beta\left(I_{M}\right)}=\ln \frac{1-\sqrt{I_{M}\left(2-I_{M}\right)} \cos \theta_{M}}{\left(1-I_{M}\right)^{2}}
$$

It is useful for application to recall that eq.10 becomes in the original units

$$
H_{M}=\omega_{e} I_{M}-\frac{\omega_{e}^{2}}{4 D_{e}} I_{M}^{2}
$$

where $0 \leq I_{M} \leq \frac{2 D_{e}}{\omega_{e}}$ 
A simple conjugate Hamiltonian for the Morse Hamiltonian is a rotor ${ }^{4} \cdot \theta_{R}$ mod $2 \pi$ is the angle variable which describes the position of the rotor with inertia momentum $J_{R}$ with the units $m_{e} / a^{2}$ defined for the above Morse oscillator. Its Hamiltonian is in action-angle variable

$$
H_{R}\left(I_{R}\right)=\frac{I_{R}^{2}}{2 J_{R}}
$$

where $p_{R}=J_{R} \dot{\theta_{R}}=I_{R}$, the variable conjugated to the angle $\theta_{R} \bmod 2 \pi$ of the rotor is also its action.

Considering the rotor $R$ as the donor $D$ and the acceptor $A$ as the Morse oscillator $M$, it is now straightforward to check from eq.10 and eq.13 that the detuning function (3)

$$
\begin{aligned}
\epsilon\left(I_{0}, I\right) & =H_{R}\left(2 I_{0}\right)-H_{R}\left(I_{0}+I\right)-H_{M}\left(I_{0}-I\right) \\
& =\frac{1}{2}\left(I_{0}-I\right)\left(3 \frac{I_{0}}{J_{R}}+I_{0}-2+I\left(\frac{1}{J_{R}}-1\right)\right)
\end{aligned}
$$

is identically zero for $J_{R}=1$ and $I_{0}=1 / 2$. In arbitrary units, this condition becomes

$$
J_{R}=\frac{m_{e}}{a^{2}}=\frac{2 D_{e}}{\omega_{e}^{2}}
$$

TET is expected to occur for a coupled rotor-Morse oscillator system when $J=1$ at any small coupling (which does not average to zero over the fast variable).

$$
\mathcal{H}=\frac{p^{2}}{2}+\frac{1}{2}\left(1-e^{-u}\right)^{2}+\frac{p_{R}^{2}}{2}+\lambda f\left(u, p, \theta_{R}, p_{R}\right)
$$

The TET solutions can be analytically calculated for special choice of the coupling energy $f\left(u, p, \theta_{R}, p_{R}\right)$ in Hamiltonian (1) which depends only on the displacement $u$ and the rotor angle $\theta_{R}$. It is physically reasonable that the coupling energy becomes independent of $u$ at large $u$ that is when the chemical bond is dissociated and to keep only the first harmonics of the $\theta_{R}$ dependence. A convenient choice is

$$
f\left(u, p, \theta_{R}, p_{R}\right)=\left(\lambda e^{-u}+b\right) \cos \theta_{R}
$$

As we shall see in the following, it is essential for chemical dissociation that the pinning potential persists for large positive $u$ and for that purpose we introduce the small constant $b \neq 0$ as a perturbation. Then, the rotor becomes

\footnotetext{
4 Note that there are other oscillators conjugate to the Morse oscillator. Their Hamiltonian should nevertheless be identical in action-angle variables in the range of energy transfer. For example, a particle oscillating in a square potential could be conjugate to the Morse oscillator as well.
} 
a pendulum where the coefficient $b+\lambda e^{-u}$ of the cosine potential depends on the displacement $u$ of the Morse oscillator and on $b$. The dynamics of the system is described by

$$
\begin{aligned}
& \ddot{u}+\left(1-e^{-u}\right) e^{-u}-\lambda e^{-u} \cos \theta_{R}=0 \\
& \ddot{\theta}_{R}-\left(\lambda e^{-u}+b\right) \sin \theta_{R}=0
\end{aligned}
$$

It is more convenient to express $u$ in action-angle variable using Eq.11

$$
\lambda e^{-u}+b=b+\frac{\lambda \cos ^{2} \beta}{1-\sin \beta \cos \theta_{M}}=\sum_{n \geq 0} a_{n}\left(I_{M}\right) \cos n \theta_{M}
$$

where

$$
\begin{aligned}
& a_{n}\left(I_{M}\right)=2 \lambda \cos \beta \tan ^{n} \frac{\beta}{2}=2 \lambda\left(1-I_{M}\right)\left(\frac{I_{M}}{2-I_{M}}\right)^{n / 2} \quad \text { for } \quad n>0 \\
& \text { and } \quad a_{0}=b+\lambda \cos \beta=b+\lambda\left(1-I_{M}\right)
\end{aligned}
$$

Then Hamiltonian (16) becomes

$$
\mathcal{H}=\frac{1}{2} I_{R}^{2}+I_{M}-\frac{1}{2} I_{M}^{2}+\sum_{n \geq 0} a_{n}\left(I_{M}\right) \cos n \theta_{M} \cos \theta_{R}
$$

Defining $\theta_{0}=\theta_{R}+\theta_{M}$ as the fast angle variable and $\theta=\theta_{R}-\theta_{M}$ the slow angle variable and their corresponding conjugate variables $I_{0}=\left(I_{R}+I_{M}\right) / 2$ and $I=\left(I_{R}-I_{M}\right) / 2$, (17) can be averaged with respect to the fast variable $\theta_{0}$ which yields the effective coupling

$\lambda C\left(I_{0}, I, \theta\right)=<\left(b+\lambda e^{-u}\right) \cos \theta_{R}>_{\theta_{0}}=\frac{a_{1}\left(I_{M}\right)}{2} \cos \theta=\lambda\left(1-I_{M}\right) \sqrt{\frac{I_{M}}{2-I_{M}}} \cos \theta$

where $I_{M}=I_{0}-I$.

Then the average Hamiltonian (2) close to TET where $I_{0}=1 / 2$ reduces to

$$
\mathcal{H}_{a}=I_{0}+\left(2 I_{0}-1\right) I+\lambda\left(1-I_{0}+I\right) \sqrt{\frac{I_{0}-I}{2-I_{0}+I}} \cos \theta
$$

When $I_{0}=1 / 2$, the Hamilton equations are

$$
\begin{aligned}
& \dot{I}=\lambda F(I) \sin \theta \\
& \dot{\theta}=\lambda F^{\prime}(I) \cos \theta
\end{aligned}
$$


where $F(I)=(I+1 / 2) \sqrt{1-2 I} / \sqrt{3+2 I}$. Since the energy of the system is conserved, its energy is the initial energy of the rotor. At TET $\mathcal{H}_{a}=$ $H_{R}(1 / 2)=1 / 2$ implies the coupling energy remains zero which yields $\theta=\pi / 2$ $\bmod \pi$. We choose $\theta=-\pi / 2$ (the other solution $\theta=\pi / 2$ is obtained by time reversal). Then $I$ obeys the simple ODE

$$
\dot{I}=-\lambda F(I)=-\lambda\left(\frac{1}{2}+I\right) \sqrt{\frac{1-2 I}{3+2 I}}
$$

This equation has two trivial time independent solutions which correspond to the zeros $I= \pm 1 / 2$ of $F(I)$. However, it can be checked on the original eqs. 18 and 19 that $I(t) \equiv 1 / 2$ or $\dot{\theta}_{R}(t) \equiv 1$ and $u(t) \equiv 0$ does not correspond to an exact solution of the system. The other solution $I(t) \equiv-1 / 2$ corresponds to an exact solution of the initial system where the particle of the Morse oscillator $u(t) \equiv+\infty$ is immobile at infinity. Actually, since $F(I)$ has a constant positive sign, the solution $I(t)$ of Eq. 28 which is not time constant is monotone decreasing and varies from $I=1 / 2$ to $I=-1 / 2$. This solution can be obtained with the change of variable $v=\sqrt{1-2 I} / \sqrt{3+2 I}$, or $I=2 /\left(1+v^{2}\right)-3 / 2$. Then, Eq. 28 yields

$$
\left(\frac{1}{1+v^{2}}+\frac{1}{1-v^{2}}\right) \dot{v}=\frac{\lambda}{2}
$$

If we define the function $\Upsilon(x)$ by the implicit equation

$$
\frac{1}{2}(\arctan \Upsilon(x)+\operatorname{atanh} \Upsilon(x))=x
$$

We have $\Upsilon(x)=-\Upsilon(-x)$. For $x$ small we have $\Upsilon(x) \approx x$. This function is monotone increasing with an asymptote $\Upsilon(+\infty)=1$. More precisely, we have for $x \rightarrow+\infty, \Upsilon(x) \approx \tanh \left(2 x-\frac{\pi}{4}\right) \approx 1-2 e^{-4 x+\pi / 2}+\ldots$

Then, $v(t)=\Upsilon\left(\frac{\lambda}{4}\left(t-t_{0}\right)\right)$ and

$$
I(t)=\frac{2}{1+\Upsilon^{2}\left(\frac{\lambda}{4}\left(t-t_{0}\right)\right)}-\frac{3}{2}
$$

$t_{0}$ is an arbitrary time we take equal to zero for simplicity. Finally, we get the time evolution of the action $I_{M}$ and the energy $H_{M}$ when initially the Morse oscillator is at rest $\left(H_{M}=0\right.$ and the rotor has initially the energy $\left.H_{R}=\frac{1}{2}\right)$

$$
\begin{aligned}
I_{M}(t) & =2 \frac{\Upsilon^{2}\left(\frac{\lambda}{4} t\right)}{1+\Upsilon^{2}\left(\frac{\lambda}{4} t\right)} \\
H_{M}(t) & =I_{M}-\frac{1}{2} I_{M}^{2}=2 \frac{\Upsilon^{2}\left(\frac{\lambda}{4} t\right)}{\left(1+\Upsilon^{2}\left(\frac{\lambda}{4} t\right)\right)^{2}}
\end{aligned}
$$




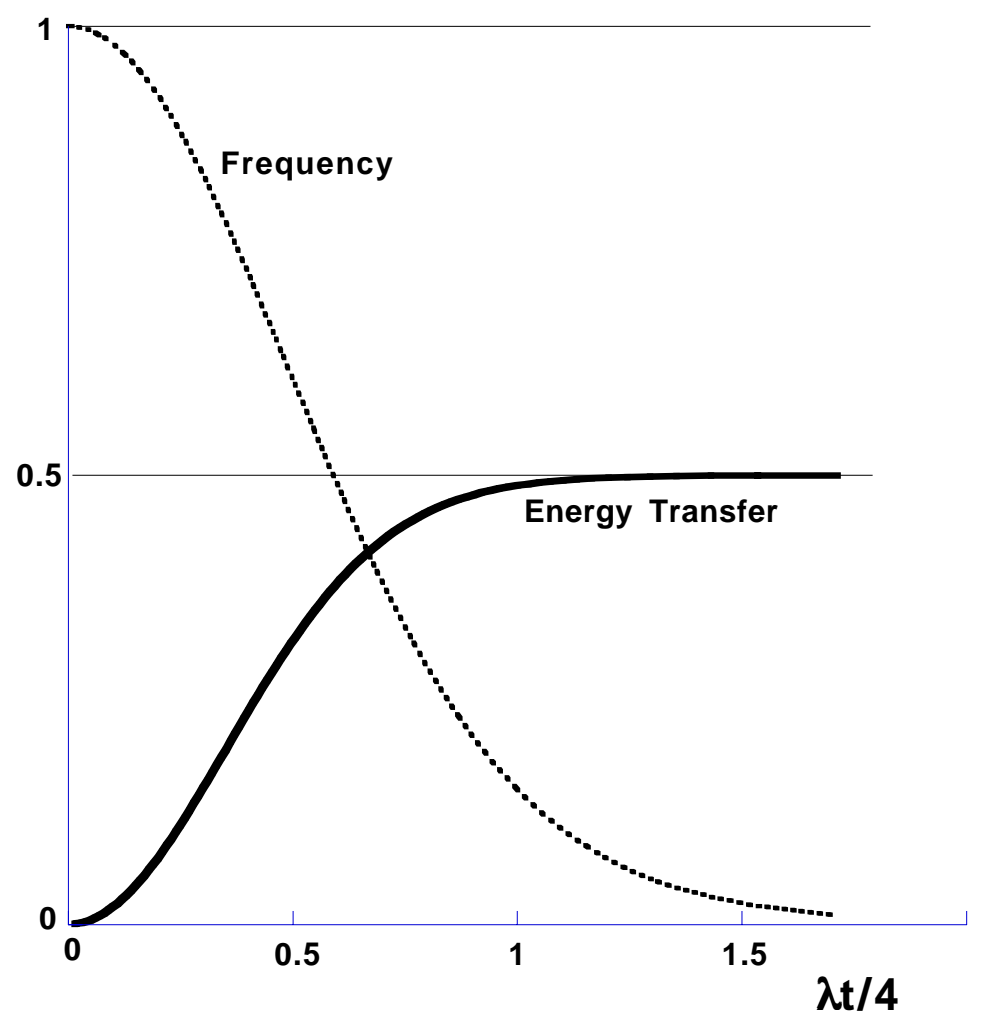

Fig. 2. Energy $H_{M}$ (full line) of the Morse Oscillator and Rotor-Oscillator frequency $\omega_{M}=\omega_{R}=1-I_{M}$ (dotted line) versus time at the integrable limit ( $\lambda$ and $b$ small $)$ as given by eq.33 and eq.32.

This TET solution corresponds to a marginal solution of an oscillator which is not time periodic. In all the examples of TET we studied before $[30,31,36]$, the energy was oscillating periodically back and forth between the donor and the acceptor oscillator. In the present case, the energy transfer to the Morse oscillator is asymptotically complete at infinite time. The consequence is that this approximate solution is not quasiperiodic and cannot correspond to a KAM torus for the initial Hamiltonian (16). As a consequence, this TET solution integrated with with the non approximate Hamiltonian should belong to a chaotic layer of the phase space and should manifest some chaotic behavior.

Our approximate Hamiltonian yields marginal chemical dissociation precisely at TET where the particle is slowly ejected at zero velocity. Fig. 2 shows the energy (33) and the frequency $\omega_{M}=1-I_{M}$ of the Morse oscillator as a function of time calculated with the approximate integrable Hamiltonian (25).

Actually, the assumption that the frequency $\dot{\theta}_{0}$ (which is twice the equal frequency of the rotor and the Morse oscillator) is fast becomes inconsistent with our result at long time. Fig. 2 shows that it goes to zero which implies that the adiabatic approximation is not valid only at the end of the transfer.

When condition (15) is fulfilled but the initial action or energy of the rotor is 
not $E_{T}=1 / 2$, complete energy transfer does not occur. The maximum $I_{m}$ of $I_{M}$ can be obtained as a function of $I_{0}$ from energy conservation. The total action is conserved $I_{R}(t)+I_{M}(t)=I_{R}(0)$ as well as the total energy

$$
\mathcal{H}_{a}=\frac{1}{2} I_{R}^{2}+I_{M}-\frac{1}{2} I_{M}^{2}+\lambda\left(1-I_{M}\right) \sqrt{\frac{I_{M}}{2-I_{M}}} \cos \theta=\frac{1}{2} I_{R}^{2}(0)
$$

which yields the equation of the trajectory on the spherical phase space

$$
\cos \theta=-\frac{1-I_{R}(0)}{\lambda} \cdot \frac{\sqrt{I_{M}\left(2-I_{M}\right)}}{1-I_{M}}
$$

The maximum value $I_{m}$ of $I_{M}$ is obtained for $\cos \theta=-1$ which yields

$$
I_{m}=1-\frac{\left|1-I_{R}(0)\right|}{\sqrt{\lambda^{2}+\left(1-I_{R}(0)\right)^{2}}}
$$

or a Lorentzian for the maximum transferred energy to the Morse oscillator

$$
E_{m}=\frac{1}{2} \frac{\lambda^{2}}{\lambda^{2}+\left(1-I_{R}(0)\right)^{2}}
$$

\section{Numerical Observations}

We now study numerically the real dynamics of the original non integrable Hamiltonian (16) and compare it with those predicted from the approximate integrable Hamiltonian (25). We use a standard Runge-Kutta method at fourth order for integrating eqs.18 and 19.

\subsection{Chemical Dissociation}

It was already noted in section 5 that since the considered TET solution of the approximate but integrable Hamiltonian (25) is marginal, it cannot be represented as a KAM torus and some chaotic behavior should manifest in the real dynamics of this solution integrated with the original Hamiltonian (16).

An example of energy transfer is shown fig.3 and its magnification fig.4 in the ideal situation for TET where $\lambda$ is small, $J_{R}=1 / 2, I_{0}=1 / 2$ and the Morse oscillator is initially at rest. We observe within a time of the order of the expected time for transfer $t_{c} \approx 2 / \lambda$ (see fig.2) that most the energy of the rotor is indeed transferred to the Morse oscillator (see fig.4). Moreover, the fit 
with the expected theoretical result (33) is excellent discarding the expected small amplitude oscillations due to the fast variable $\theta_{0}$.

However, we do not observe (immediately), the expected marginal chemical dissociation predicted by formula (33) at large time. Instead of, the energy of the Morse oscillator partially returns to the rotor and next oscillates between the rotor and the Morse oscillator with irregular amplitudes. Small perturbations of the initial conditions change the amplitudes and the number of these oscillations which thus can be considered as chaotic.

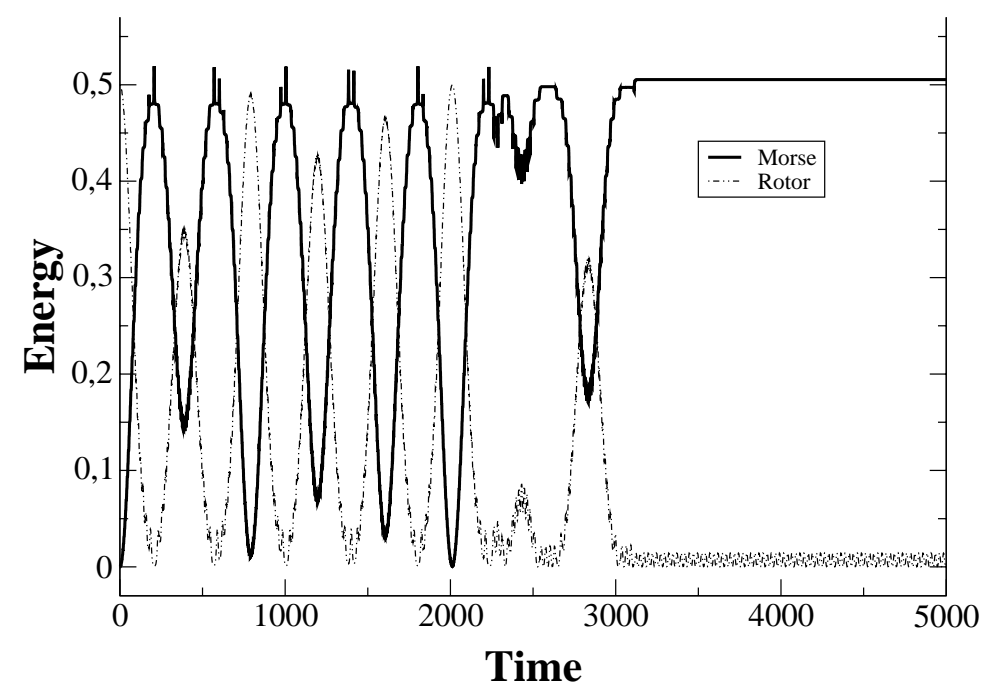

Fig. 3. Rotor energy $H_{R}=\dot{\theta}_{R}^{2} / 2$ (dotted-dashed line) and Morse oscillator energy (8) $H_{M}(p, u)$ (full line) versus time obtained from the dynamical eqs.18 and 19 at $b=\lambda=2.10^{-2}$ and the initial conditions for TET $\left(\dot{\theta}_{R}(0)=1, \theta_{R}(0)=\pi / 2\right.$, $u(0)=\dot{u}(0)=0)$.

However, this chaotic manifestation is only transient. After a certain number of oscillations (which is not well determined because it depends on small perturbation of the initial conditions), the particle of the Morse oscillator is ejected at infinity with a finite velocity which also is not well determined. Fig. 5 shows the rotor angle versus time in the last stage before ejection where $u(t) \rightarrow+\infty$. The angle variation slows down but instead of stopping to an asymptotic value, the rotor angle continue to oscillate periodically in time in some angle interval $[(0 \bmod \pi)-\delta,(0 \bmod \pi)+\delta]$ where $0<\delta<\pi / 2$. Indeed, when $u(t)$ goes to infinity, the coupling energy goes to $b \cos \theta_{R}$ which implies that the rotor is still submitted to a small angular dependent potential and thus is a pendulum.

If the rotor goes at rest $\left(\theta_{R}=\pi\right.$ when $b<0$ or 0 when $\left.b>0\right)$ when the particle of the Morse potential is at $+\infty$, the coupling energy becomes negative and 


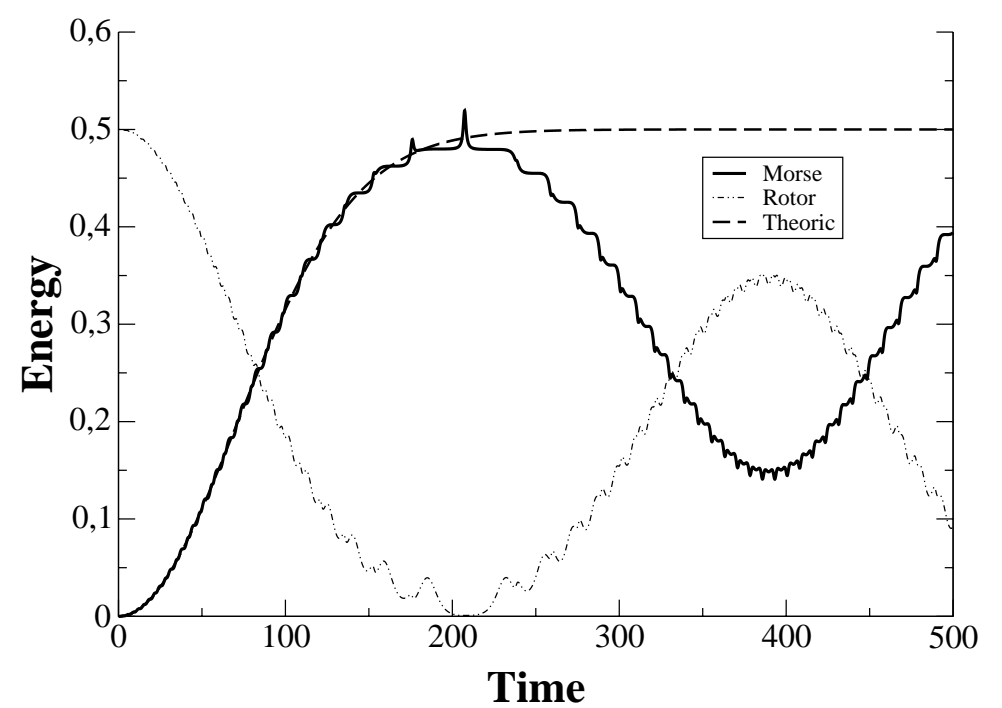

Fig. 4. Magnification of fig.3 at short time (full line and dotted-dashed line) and fit with eq.33 (dashed line).

equal to $-|b|$. Since the potential energy of the particle in the Morse potential is just $1 / 2$ the initial energy of the rotor, energy conservation requires that the kinetic energy of the particle of the Morse potential is $\dot{u}^{2} / 2=|b|$ and constant. As a result, the particle of the Morse potential is ejected at infinity with a finite velocity. The same result holds if the rotor stays dangling in its pinning potential $b \cos \theta_{R}$ with a negative energy that is with an angle which oscillates in an interval with half width $\delta<\pi / 2$. Actually, the kinetic energy of the ejected particle or the pinning energy of the rotor appears numerically as random variables. It is also worthwhile to note that when $b=0$ and the initial energy is $1 / 2$, we numerically checked that there is no dissociation at all and that the chaotic behavior is not transient but persists forever.

We observe for moderately large value of the coupling parameter $\lambda$, that there is a nonvanishing interval for the initial energy of the rotor around the ideal value for TET, where the atom in the Morse potential is finally ejected at infinity with some nonzero kinetic energy. This energy is borrowed to the pinning energy of the rotor in the residual potential due to the coupling. Then, at the final stage of the dynamical evolution of the system, the rotor oscillates periodically around its equilibrium position while the particle in the Morse potential escape at infinity with a constant velocity.

\subsection{Interpretation}

These features can be easily interpreted because they are commonly observed in perturbed integrable systems such as $2 \mathrm{~d}$ nonintegrable discrete maps. It 


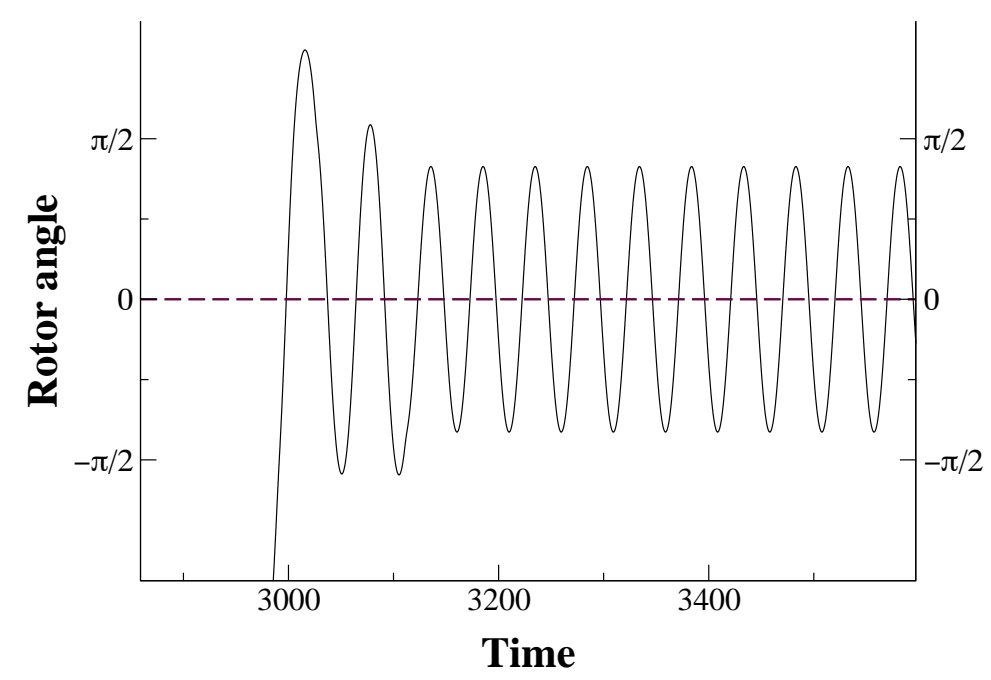

Fig. 5. Rotor angle $\theta_{R}(t)$ versus time in the vicinity of chemical dissociation shown fig.3.

is well-known that many quasiperiodic trajectories persist as KAM tori but not those which are resonant or almost resonant which break up and generate stochastic layers. Separatrix ( such as the marginal TET trajectory in our case) also generate chaotic layers.

Unbound stochastic layers are also common in unbounded maps. They can be found for example in the map $x^{\prime}=x^{3}+a x-y, y^{\prime}=x$ where $|a|<2$ ). There is a central island made of concentric KAM tori with thin bounded stochastic layers in between and a peripheral stochastic layer which extends at infinity. This region surrounds the central KAM tori but also many smaller KAM islands.In that region, the trajectories are unbound with probability 1. There are trajectories which are bounded at all (discrete) time close to the KAM tori but they are known to have zero measure and are unstable. Thus, most initial conditions (with probability one) even close to the KAM tori yield trajectories which finally escape to infinity. Their dynamics may exhibit initially some almost regular oscillations reminiscent of the nearby KAM orbits but after some number of iterations they becomes unstable and escape at infinity and never return.

In our case, because of energy conservation, the projection of the stochastic layer in the plane $(\dot{u}, u)$ does not extend at infinity in all directions. It is necessarily confined to a certain domain. If the rotor initial energy is $1 / 2$, we readily get the inequality on the Morse oscillator energy

$$
H_{M}(t)<\frac{1}{2}+|b|
$$

. When $b$ is non zero, this domain extends to infinity along the $u$ axis in a stripe with nonvanishing thickness which determines a window where the trajectories can escape. It turns out that when $|b|$ is nonzero, this stochastic layer which 
is inside that domain is also unbound and extends at infinity. The sensitivity to the initial conditions (at constant energy) for example the initial phase of the rotor is clearly a consequence the chaotic behavior in this stochastic layer.

When $b=0$ the probability to escape for the TET trajectory is zero because the escaping window shrink to zero at infinity and we observe only chaotic oscillations. When $b \neq 0$ this window opens and the probability to escape is 1 but after a certain number of transient chaotic oscillations. The probability that this number of oscillation be infinite, and thus that the particle does not escape is zero.

When the TET condition is not fulfilled, for example when varying the initial energy of the rotor from $1 / 2$, the approximate integrable Hamiltonian (25) yields quasiperiodic trajectories with incomplete energy transfer instead of a marginal trajectory. These trajectories may survive as a KAM tori or in case of resonance as thin stochastic layers imbedded between these KAM tori. In both cases, the trajectories become bounded and there is no chemical dissociation. This is precisely what is observed when the initial rotor energy get out the narrow window in energy around the optimal TET energy and which we describe next.

In our case, the trajectories close in energy to the TET energy, are well described by the integrable approximate Hamiltonian (25) while the averaged fast variable $\theta_{0}$ is really fast and decouples from the slow variables. The chaotic behavior of our system essentially originates from the fact this fast variable may become slow. This situation occurs when the energy transfer is close to be complete, it is then not valid to the initial Hamiltonian average over $\theta_{0}$. Then, the time oscillations of $\theta_{0}$ couples to the slow variables and deviates the real trajectory from the approximate integrable trajectory. But this deviation should depend on the phase of the oscillations of $\theta_{0}$ (see the small amplitude oscillations visible on fig.3). Depending on the phase of the oscillation of $\theta_{0}$ which can be assumed to be random, the particle in the Morse oscillator may be either kicked out with some velocity or bounced back. Then, the real trajectory borrows another periodic integrable trajectory, then comes back almost in the same configuration but with another phase for $\theta_{0}$, again may either escape with some probability or bounce back, and so on...

\subsection{Energy Selectivity}

Energy selectivity is an aspect of TET essential for its potential applications. We analyze this selectivity in the Rotor-Morse oscillator model with more details. When the initial energy of the rotor is not precisely $H_{R}(1 / 2)=1 / 2$, the approximate Hamiltonian (25) yields a maximum energy transfer $E_{m}=$ 
$\max _{t} H_{M}(t)$ (given by eq.37) which is not complete. Then only a part of the initial energy periodically oscillates between the rotor and the Morse oscillator. The maximum energy transfer given by eq.37 is a Lorentzian curve.

Fig.6 compares this result with the numerical calculation from the original Hamiltonian (16) of the maximum energy transferred to the Morse oscillator as a function of the initial action $I_{R}(0)=\dot{\theta}_{R}(0)$ (or frequency) of the rotor $\left(\max _{t} H_{M}(t)\right.$ is measured over numerical times which are long enough to be insensitive)

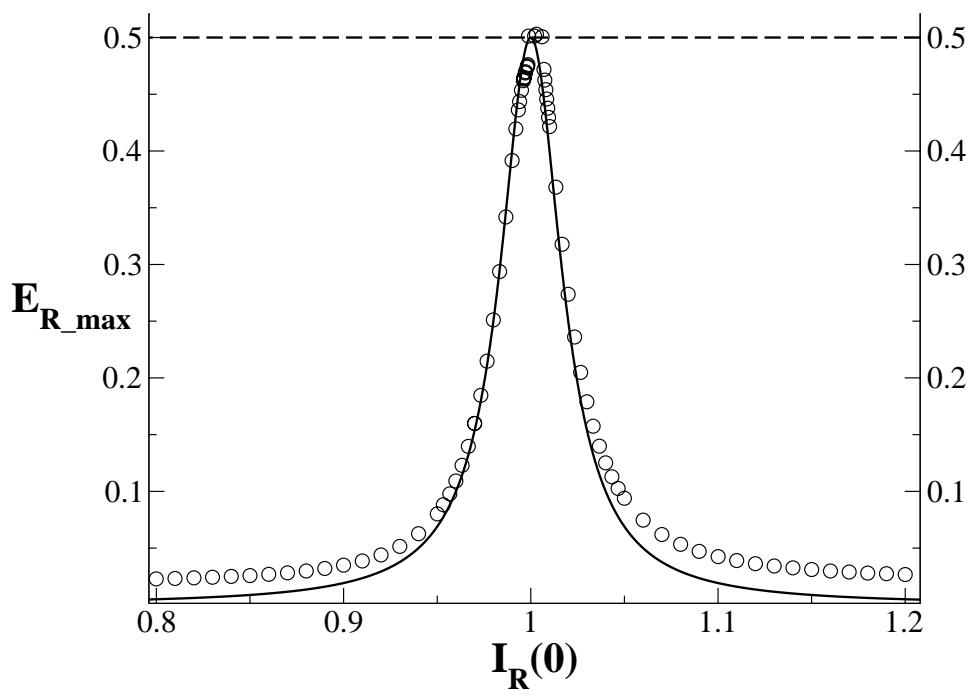

Fig. 6. Maximum energy transfer on the Morse oscillator calculated numerically versus initial frequency $\dot{\theta}_{R}(0)$ of the rotor (open dots)compared with the analytical prediction (37) (full line) (same parameters as fig.3) and $\theta_{R}(0)=\pi / 2, u(0)=\dot{u}(0)=0$.

The numerical results are globally in good agreement with the theoretical predictions except for some interesting new features we now describe.

- Most numerical values of the maximum energy transfer plotted versus the initial velocities of the rotor $\dot{\theta}_{R}(0)$, appear to be distributed on a smooth peaked curve which is strictly below the dissociation energy $1 / 2$ of the particle in the Morse potential. However, there is a narrow interval for $\dot{\theta}_{R}(0)$ close to the ideal initial condition $\dot{\theta}_{R}(0)=1$ for TET (see magnification of fig.7) where the maximum energy transferred to the Morse oscillator discontinuously jumps to values strictly larger than the dissociation energy $1 / 2$. Actually, with these initial conditions on the rotor, we have chemical dissociation that is the particle in the Morse potential never returns to its original position but is ejected at $+\infty$. Its velocity becomes constant and its kinetic energy is just the excess of transferred energy over $1 / 2$. This kinetic energy was carefully analyzed as a function of $I_{R}(0)=\dot{\theta}_{R}(0)$ varying by very small steps. It clearly appears to be a randomlike variable sharply dependent on the most tiny perturbations of the initial conditions (see the magnification of the tip of the TET peak fig.7). 


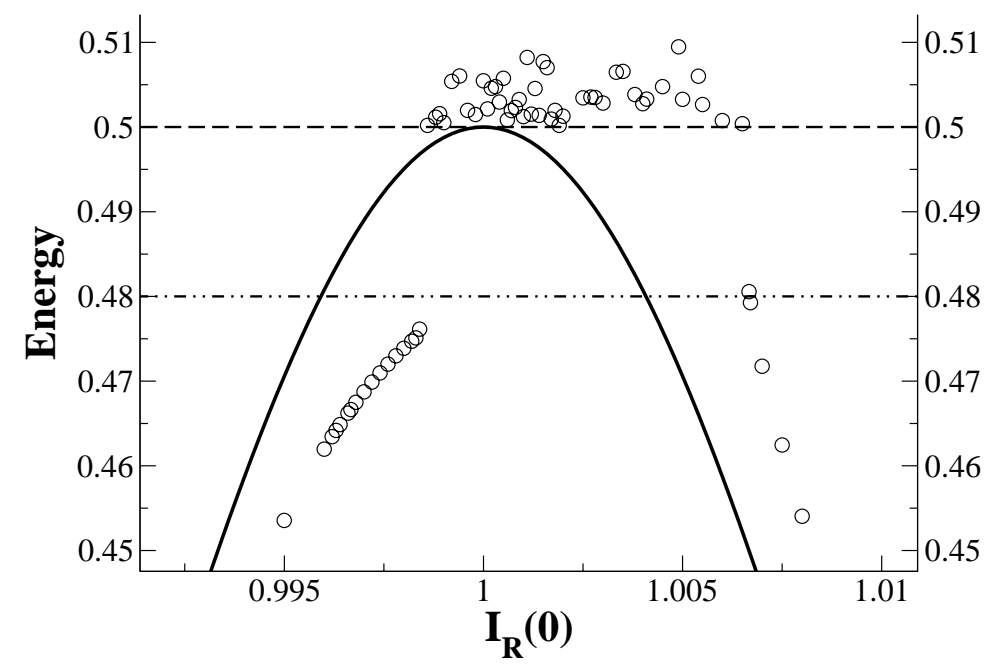

Fig. 7. Magnification of fig.6 at the peak.

According to the above interpretation, the unbounded stochastic layer generating dissociation should persist in a small neighborhood of the ideal initial energy for TET

- The initial conditions which do not produce chemical dissociation corresponds to the smooth part of the maximum energy transfer curve (6). Then, the energy slowly and periodically oscillates between the rotor and the Morse oscillator while the global dynamics of the rotor-Morse system with hamiltonian (16) looks regular and quasiperiodic.

This feature also should be expected because when the perturbation on the initial energy becomes large enough, the quasiperiodic trajectory of the approximate Hamiltonian survives either as a KAM torus or in case of resonance as a thin bounded stochastic layers imbedded within KAM tori. There is no more unbounded stochastic layer and thus no chemical dissociation.

However, we note small discrepancies between the numerical datas and their analytical fit (37).

- The peak magnification of fig.7 shows that the tip of the smooth (truncated) peak referred to the theoretical curve is shifted to the right hand side that is to larger values of $I_{R}(0)=\dot{\theta}_{R}(0)$.

- The intermediate part of the peak corresponding to a substantial maximum energy transfer intermediate between 0 and $1 / 2$ (that is most of the visible curve fig.6) is well fitted with formula (37)

- far away from top of the TET peak that is in the tails of the Lorentzian fit where the rotor and the Morse oscillator are out of resonance, the observed value of maximum energy transfer is systematically larger than those predicted although both values are small.

We can explain qualitatively these features. 
We noted above that when the energy transfer to the Morse oscillator approaches its dissociation energy, the equal frequencies of the rotor and the Morse oscillator goes to zero and thus the angle variable $\theta_{0}$ which was assumed to be the unique fast variable of the system becomes slow. Consequently, the nonintegrable hamiltonian (16) is not well approximate by the integrable Hamiltonian (25). and a chaotic behavior appears in the long time scale variations of amplitude of the energy oscillations between rotor and Morse oscillator. This situation corresponds to the dynamics at the top of the peak fig.7.

Another situation where the initial hamiltonian (16) is not well approximate by (25) but for a different reason, is obtained when the rotor and the Morse oscillator are far from resonance. This situation occurs in the tail of the peak fig.6. That situation does not involve any substantial chaotic behavior since it is well-known that KAM theory applies for the non resonant quasiperiodic trajectories of the rotor-Morse oscillator system which is integrable at zero coupling and where the coupling is a small perturbation.

Finally, it turns out that the approximation of (16) by (25) is accurate only in the intermediate regime between chemical dissociation and a small energy transfer that is just where the numerical datas fig.6 well agree with their analytical fit.

Actually, a standard perturbation theory applied to the initial Hamiltonian (16) should determine in principle through canonical transformations a series of approximate integrable Hamiltonians $\mathcal{H}_{p}$ at successive order starting from $\mathcal{H}_{1}=\mathcal{H}_{a}(25)$. For example, the off-diagonal terms of Hamiltonian (23) which were averaged to zero with the assumption of fast variable $\theta_{0}$, should contribute as a higher order correction of the approximate lowest order Hamiltonian (25). We shall not perform here this tedious calculation. In the absence of chaos, that is when the dynamics of the system is quasiperiodic on a KAM torus, this series of approximate integrable Hamiltonian $\mathcal{H}_{p}$ is convergent and describe the perturbations of KAM torus. The consequence is that the detuning function (3) is still a well defined function although it has to be renormalized.

We expect that these corrections should explain the disagreement between the fit (37) and the numerical datas of fig. 6 and 7 which are observed in the smooth part of the curve mostly close to the tip of the peak and in the tails for the above reasons. In particular, the optimum value of $I_{0}$ for complete TET should shifted as observed close to the top of the numerical peak of TET.

We can make a rough estimation of the width of the window of initial energies where we expect to have chemical dissociation on the basis of empirical arguments. The trajectory are well described by the approximate Hamiltonian (25) while the averaged fast oscillation of $\theta_{0}$ is really fast. Actually, the coupling 
energy (17) initially depended on $\theta_{0}$ before averaging. When the amplitude $u$ of the Morse oscillator is large and thus when the variation of $\theta$ and $\theta_{0}$ are both slow, it becomes $b \cos \theta_{R}=b \cos \left(\theta+\theta_{0}\right) / 2$. Although this energy would be zero on time average, it is not zero at a precise time and depends on the phase of the rotor $\theta_{R}$.

Consequently, when a trajectory close to TET reaches its maximum of energy transfer $E_{m}$ which is expected to be (37) calculated with the approximate integrable Hamiltonian (25), energy conservation considered for the non approximate Hamiltonian requires that the real energy of the Morse oscillator at this maximum is slightly different and equal to $E_{m}-b \cos \theta_{R}$ when $\theta_{R}$ slowly vary. This effect is in some sense equivalent to a kick on the integrable trajectory which depends depends on the rotor phase. If we have $E_{m}-b \cos \theta_{R}>1 / 2$, then, the particle in the Morse potential has enough energy for escaping and its kinetic energy is then $E_{m}-1 / 2-b \cos \theta_{R}$ (note the coupling force between the Morse oscillator and the rotor vanishes at large $u$ ). If we have $E_{m}-b \cos \theta_{R}<1 / 2$, then the particle bounces back. It starts a new oscillation well described again by the integrable Hamiltonian (25) but with a small change of initial conditions. The trajectory return again at the same maximum amplitude but with another value for $\theta_{R}$, again the particle may dissociate or bounce back and so on... Considering the values of $\theta_{R}$ at the maxima of amplitude becomes random, this model considers the transient chaotic behavior observed for the unbounded stochastic layer as generated by small random kicks at its maximum amplitudes.

According to this description, when the maximum energy (37) transferred to the Morse potential plus the maximum pinning energy $|b|$ of the rotor, is larger than the dissociation energy $1 / 2$, Then, we obtain that chemical dissociation occurs when $1-\delta<I_{R}(0)<1+\delta$ where $\delta^{2}=2\left|b \lambda^{2}\right| /(1-2|b|) \approx 2\left|b \lambda^{2}\right|$ for $|b|$ small. Thus for the example of fig.7 where $b=\lambda=2.10^{-2}$, we obtain $2 \delta \approx 8.10^{-3}$ which roughly corresponds to the observed peak width.

\subsection{Absence of Chemical Dissociation at Large Coupling}

At large coupling, the energy landscape of the model is obviously strongly affected. However, the dissociation energy is unchanged. It is interesting to test again if chemical dissociation still may occur in equivalent conditions that is with the dissociation energy $1 / 2$ as initial energy and the same pinning energy $|b|$ (which yields the maximum kinetic energy to the particle). We observe that chemical dissociation disappear. 
The energy flux received by the Morse oscillator is

$$
\frac{d H_{M}}{d t}=\frac{d H_{M}}{d I_{M}} \dot{I}_{M}=-\left(1-I_{M}\right) \frac{\partial \mathcal{H}}{\partial \theta_{M}}
$$

where $\mathcal{H}$ is given by eq.23. Then,

$$
\frac{d H_{M}}{d t}=\lambda\left(1-I_{M}\right) \sum_{n \geq 0} a_{n}\left(I_{M}\right) n \sin n \theta_{M} \cos \theta_{R}
$$

When averaging over the fast variable $\theta_{0}$, we obtain the average energy flux

$$
<\frac{d H_{M}}{d t}>\approx \frac{\lambda}{2}\left(1-I_{M}\right) a_{1}\left(I_{M}\right) \sin \theta
$$

When we have perfect TET, the phase difference between the rotor and the Morse oscillator is $\theta=\theta_{R}-\theta_{M} \approx \pi / 2$. The perfect phase coherence between the rotor and the Morse oscillator maintains the energy flux positive at its maximum value all along the transfer. Thus, at small coupling $\lambda$, the situation of targeted energy transfer corresponds to the fastest possible energy transfer between the rotor and the Morse oscillator.

When the coupling $\lambda$ increases, $I_{M}, I_{R}$ and $\theta$ are not slow variables anymore, so that the integrable hamiltonian (25) obtained by averaging over the unique fast variable $\theta_{0}$ becomes a poor approximation of (16).

We numerically tested the behavior of the system with the same initial conditions $\left(\theta_{R}(0)=\pi / 2, \dot{\theta}_{R}(0)=1, u(0)=\dot{u}(0)=0\right)$ when increasing $\lambda$ from small values while keeping the available energy $|b|$ for dissociation constant.

Actually, we observe initially that when $\lambda$ increases from small values, the characteristic time for energy transfer decreases proportionally to $1 /|\lambda|$ as expected from eq.33 and subsequently chemical dissociation rapidly occurs after a short chaotic transient of few energy oscillations as shown fig.3. However at larger values when $\lambda$ approaches from below a critical value $\lambda_{c} \approx 0.77$, the duration of the chaotic transient sharply increases. The fast chaotic energy oscillations becomes less and less efficient for producing the chemical dissociation. This effect is clearly due to the fact that the trajectory becomes less chaotic (i.e. its Lyapounov exponent goes to zero) and tends to be quasiperiodic. For $\lambda>\lambda_{c}$, the trajectory of the system becomes bounded and perfectly quasiperiodic. It is then clear that chemical dissociation will never occur even over very long time.

We demonstrate this behavior by exhibiting a Poincaré map of the dynamics of the system. We consider the $2 \mathrm{D}$ manifold $\mathcal{M}$ imbedded in the $4 \mathrm{D}$ phase space $\left(\theta_{R}, p_{R}=\dot{\theta}_{R}, u, p=\dot{u}\right)$ defined by $\theta_{R}(0)=\pi$ modulo $2 \pi, \dot{\theta}_{R}(0) \geq 0$ and 


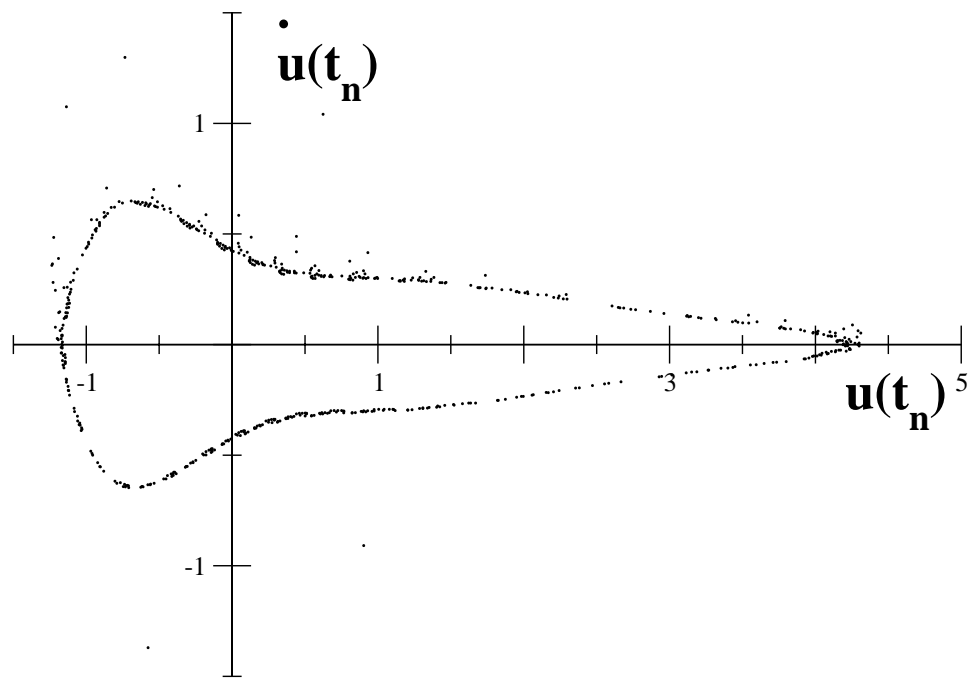

Fig. 8. Projection of the sequence of points $P_{n}=P\left(t_{n}\right)$ in the plane $(u, \dot{u})$ generated from the trajectory with initial conditions $\left(\theta_{R}(0)=\pi / 2, \dot{\theta}_{R}(0)=1, u(0)=\dot{u}(0)=0\right.$ for $\lambda=0.764$ and $b=0.02$.

the total energy $(16) \mathcal{H}\left(\theta_{R}, p_{R}, u, p\right)=\frac{1}{2}$ corresponding to the initial energy at TET. Any point $P$ in this manifold can be represented uniquely by its projection in the $2 \mathrm{D}$ plane $\mathbf{P}_{M}(P)=(u, \dot{u})$. This projection is restricted to the domain $\mathcal{D}$ defined by

$$
\frac{1}{2} \dot{u}^{2}(0)+V_{M}(u(0)) \leq \frac{1}{2}
$$

We consider the return map $\mathbf{F}(P): \mathcal{M} \rightarrow \mathcal{M}$ as the first point which returns in $\mathcal{M}$ for the continuous trajectory $P(t)=\left(\theta_{R}(t), \dot{\theta}_{R}(t), u(t), \dot{u}(t)\right)$ generated with the evolution eqs. 18 and 19, by the initial conditions

$$
P(0)=\left(\theta_{R}(0)=\pi / 2, \dot{\theta}_{R}(0)=\sqrt{1-V(u(0))-\dot{u}^{2}(0)}, u(0), \dot{u}(0)\right)
$$

Defining the discrete monotone increasing sequence of times $t_{n}$ by the condition $\theta_{R}\left(t_{n}\right)=\pi$ modulo $2 \pi$ and $\dot{\theta}_{R}\left(t_{n}\right)>0$, then we obtain recursively $\mathbf{F}\left(P\left(t_{n+1}\right)\right)=P\left(t_{n}\right)$. We plot the projection of this sequence of points of the sequence of points $P_{n}$ which have coordinates $\left(u\left(t_{n}\right), \dot{u}\left(t_{n}\right)\right)$. Since $d \theta_{R}(0) \wedge$ $d p_{R}(0)+d u(0) \wedge d p(0)=d \theta_{R}\left(t_{1}\right) \wedge d p_{R}\left(t_{1}\right)+d u\left(t_{1}\right) \wedge d p\left(t_{1}\right)$ and since $\theta_{R}(0)=$ $\theta_{R}\left(t_{1}\right)=\pi$ is fixed, we have in $\mathcal{M}, d u(0) \wedge d p(0)=d u\left(t_{1}\right) \wedge d p\left(t_{1}\right)$, which implies the projected return map of $\mathbf{F}$ in $\mathcal{D}$ is symplectic (i.e. area preserving in $2 \mathrm{D})$.

Figs. 8 and 9 show two examples of the obtained distribution of points $P_{n}$. Fig.8 is obtained for $\lambda$ smaller but close to $\lambda_{c}$. The distribution of points $P_{n}$ projected in $\mathcal{D}$ is still clearly erratic although they tend to cluster around a closed curve. 


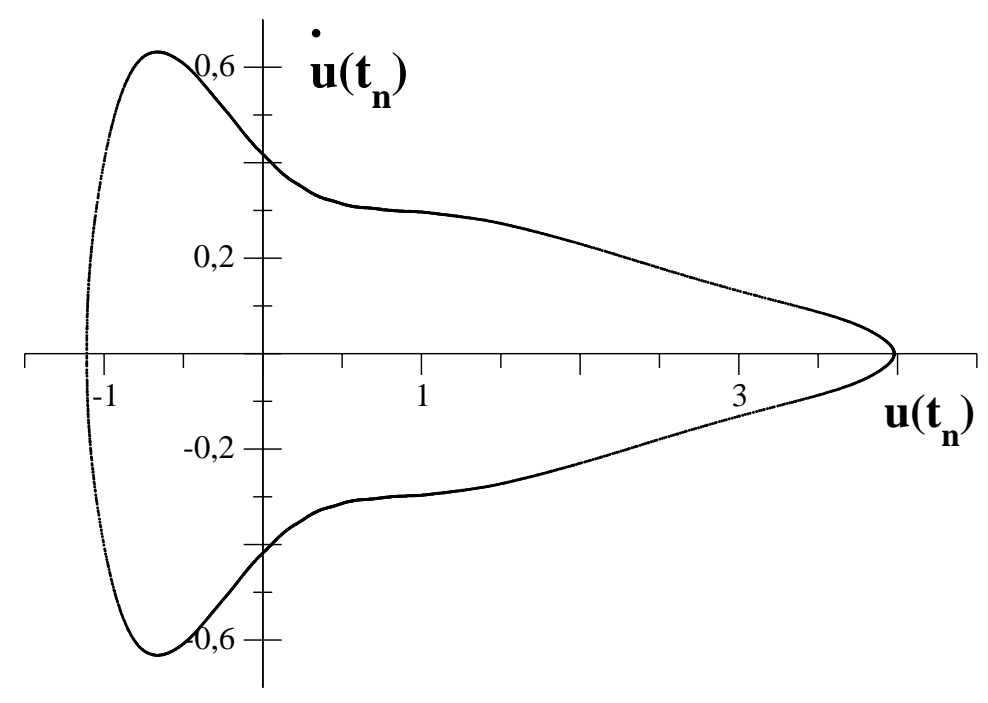

Fig. 9. Same as fig. 8 but for $\lambda=0.78$.

The existence of the unbounded stochastic layer is clearly visualized. Actually, the number of points $P_{n}$, which appears within the bounded frame shown in the figure, is finite because for $n$ large enough $P_{n}$ escapes to infinity but however it is large. Indeed, when the particle escapes at infinity from the Morse potential, the rotor angle oscillates periodically around the value 0 modulo $2 \pi$ and does not reach the value $\pi$ anymore. Thus, the number of points appearing within the frame is small when $\lambda$ is small because chemical dissociation occurs after few energy oscillations. When $\lambda$ approaches $\lambda_{c}$ from below, this number grows and becomes very large as can be seen on fig8. It diverges at $\lambda_{c}$.

Fig.9 which is obtained for $\lambda$ larger and close to $\lambda_{c}$, shows that within the dot size accuracy, the number of points in the frame becomes apparently infinite. Their distribution is dense and uniform on a thin smooth closed curve. This result implies that this curve is invariant by the Poincaré map transformation $\mathbf{F}$ and thus is the section of an invariant KAM torus by the manifold $\mathcal{M}$. The trajectory of the system for this initial condition is quasiperiodic and bounded and the Poincaré map $\mathbf{F}$ is homeomorphic to a rotation on a circle with an angle incommensurate with $2 \pi$. As a result, no chemical dissociation can occur in that case.

In conclusion, it appears that ultrafast chemical dissociations by TET can operate efficiently only when the coupling is not too large. There is an optimal coupling where chemical dissociation becomes faster. 


\section{Some Comments on Quantum aspects}

It was shown on some examples in [36], that TET may persist in quantum models when a sufficiently large number of quanta is involved, and that the classical behavior is already well recovered for a relatively small number of quantas. Then, it corresponds to the tunneling of high level excitation from the donor oscillator to the acceptor. Actually, this transfer is not direct but is done through a finite sequence of almost degenerate intermediate quantum states connecting the states with a maximum energy on the Donor and on the Acceptor respectively (these degeneracies appear through semiclassical quantization of the classical system when the detuning function (3) vanishes). However, no chemical dissociation was involved in the studied example where the energy wave packet was only oscillating back and forth with however a slow defocusing in the quantum case.

Considering the rotor-Morse oscillator model as quantum, it is supposed to deal with large amount of energy involving many quanta of energy and we may believe to be in proper conditions where the classical approximation should be good. However, we are in a situation where the chaotic behavior appeared to be essential for chemical dissociation in the classical case. The question is to know if their will be quantum manifestations of this classical chaos which are essential or if the quantum dissociation simply reduces to a tunnelling problem.

With that respect, it is interesting to note that our rotor-Morse model can be easily quantized in the uncoupled case $(\lambda=0)$. The eigenenergies of the bound states of the quantum Morse oscillator with potential (7) are exactly known $[37,38]$

$$
E_{n}^{(M)}=\left(n+\frac{1}{2}\right) \hbar \omega_{e}-\left(n+\frac{1}{2}\right)^{2} \frac{\hbar^{2} \omega_{e}^{2}}{4 D_{e}}
$$

The Morse potential has a finite number $n_{e}+1$ of bounded states $\mid n>_{M}$ where $0 \leq n \leq n_{e}$ with

$$
n_{e}=\operatorname{int}\left(\frac{2 D_{e}}{\hbar \omega_{e}}-\frac{1}{2}\right)
$$

( int denotes integer part). It can be noted that the Morse potential is one of the rare potentials where the semiclassical quantization[40] is exact since $E_{n}^{(M)}=H_{M}((n+1 / 2) \hbar)$ where $H_{M}$ is given (12). For having at least one bounded state in order the chemical bond to exist, we should have $n_{e} \geq 0$ or $4 D_{e}>\hbar \omega_{e}$. Actually, we shall assume that $n_{e}$ is substantially large (typically $n_{e}>5$ is sufficient) which is generally true for strong chemical bonds.

The quantum levels of the free rotor corresponding to the classical Hamiltonian 
(13) are

$$
E_{n}^{(R)}=n^{2} \delta_{R}
$$

where $\delta_{R}=\frac{\hbar^{2}}{2 J_{R}}$ and integer $n$ now runs from $-\infty$ to $+\infty$. The corresponding normalized eigen states are $\mid n>_{R}=e^{i n \theta_{R}} / \sqrt{2 \pi}$. Again, the semiclassical quantization is exact for the quantum rotor since $E_{n}^{(R)}=H_{R}(n \hbar)$ [40]. Note that these states are twice degenerate since $n$ can be either positive or negative. This is the quantum counterpart of the fact that in the classical TET occurs independently of the direction of rotation of the rotor.

If one consider, the energies $E_{p}^{(n)}$ of the quantum states $\left|p>_{R} \otimes\right| n-p>_{M}$ of the whole system where the Morse oscillator is in its $p$ th excited states and the rotor in its $(n-p)$ th excited state with $0 \leq p \leq n$, we have

$$
E_{p}^{(n)}=E_{p}^{(M)}+E_{n-p}^{(R)}=H_{M}\left(\left(p+\frac{1}{2}\right) \hbar\right)+H_{R}((n-p) \hbar)
$$

The discrete function

$$
\epsilon_{p}^{(Q)}=E_{0}^{(n)}-E_{p}^{(n)}=p\left(2 n \delta_{R}+\frac{\hbar^{2} \omega_{e}^{2}}{4 D_{e}}-\hbar \omega_{e}\right)+p^{2}\left(\frac{\hbar^{2} \omega_{e}^{2}}{4 D_{e}}-\delta_{R}\right)
$$

considered versus $p$ is the quantum analogous of the detuning function. When the classical condition for TET

$$
\delta_{R}=\frac{\hbar^{2}}{2 J_{R}}=\frac{\hbar^{2} \omega_{e}^{2}}{4 D_{e}}
$$

is fulfilled, we get $\epsilon_{p}^{(Q)}=p \delta_{R}\left((2 n+1)-\frac{\hbar \omega_{e}}{\delta_{R}}\right)$ which vanishes when

$$
2 D_{e}=\left(n_{e}+\frac{1}{2}\right) \hbar \omega_{e}
$$

and $n=n_{e}$ In that case, the last bounded state of the quantum Morse potential is marginally bounded.

Thus, when the rotor is chosen initially in its $n_{e}$ quantum state, there is a path of exactly degenerate quantum states which brings the Morse oscillator from its ground state to the marginal quantum state at the limit of dissociation ${ }^{5}$.

When the coupling parameter $\lambda$ is not zero but small, the Hamiltonian contains small off-diagonal terms which couples this chain of $n_{e}+1$ quantum states and further to the continuum of extended states of the quantum Morse oscillator which suggest that the tunnelling of the particle out of the Morse

\footnotetext{
5 Note however, that this is a peculiarity of our model where the semiclassical quantization is exact. In general, the quantum TET conditions involves small quantum corrections when compared to the classical one.
} 
potential should be quite easy in case of classical TET. There is no reason to believe that quantum manifestation of the classical chaos are still important for this quantum chemical dissociation. Then, classical TET should manifest as a sharp peak in the quantum yield of the chemical dissociation as a function of the initial energy and of the rotor or the Morse oscillator parameters. The maximum of the quantum yields should correspond to the situation with quasidegenerate pathway of quantum states. This is nothing but the quantum correspondence of the classical chemical expressway.

It is worthwhile to perform more analytical and numerical investigations in order to estimate precisely the quantum yields of this chemical dissociation and its selectivity.

It should be noted that because of the double degeneracy of the quantum states of the rotor and since quantum states can be linearly superposed we should also expect chemical dissociation if the initial quantum state is a arbitrary combination of the state $\left|n_{R}>_{R} \otimes\right| 0>_{M}$ and $\left|-n_{R}>_{R} \otimes\right| 0>_{M}$ The careful analysis of this quantum rotor-Morse oscillator system is left for further works.

\section{Final comments and summary}

We have introduced the concept of conjugate nonlinear oscillators or local modes which may be viewed as a nonlinear extension of the concept of linear resonance although it requires more conditions involving also the nonlinearities. Weakly coupled pair of conjugate nonlinear oscillators may exchange coherently a well defined amount of energy only (Targeted Energy Transfer).

Using this effect, a simple pedagogical model is shown to induce selectively the chemical dissociation of a selected bond. In our example the chemical bond is modelled as a Morse oscillator and the conjugate oscillator is a rotor with appropriate momentum tuned for conjugacy. For a well defined amount of energy injected to the rotor which is precisely the dissociation energy, this energy is coherently and completely transferred to the Morse oscillator and then induces chemical dissociation. This TET effect is highly selective because it can break only a bond which is conjugated with the rotor while it would let intact any other bond. Nevertheless, this model clearly needs improvements for application to real systems.

In order to fix the ideas, let us consider a physical example of rotor and the characteristics of the chemical bond it could dissociate. Biomolecules contain many methyl groups $\mathrm{C}-\mathrm{C}-\mathrm{H}_{3}$ which terminates carbon chains and forms rotors. The three protons forms a rather rigid trihedra with axis the $C-C$ bond which may rotate around its axis. They are more often moving in three 
fold symmetry potential but sometimes could be almost free depending upon its environment.

The length of the bond $C-H$ is $1.08 \AA=1.0810^{-10} \mathrm{~m}$ thus $a \approx 1.08 / \sqrt{3} \AA$ is the distance of the proton from the axis of the $\mathrm{C}-\mathrm{H}_{3}$ Rotor. The mass of the proton is $m_{p}=\frac{1}{6.02} \cdot 10^{-26} \mathrm{~kg}$. The velocity of the proton is $a \dot{\phi}$ and its kinetic energy is $\frac{1}{2} m_{p} a^{2} \dot{\phi}^{2}$. The rotor kinetic energy which involves three protons is $\frac{3}{2} m_{p} a^{2} \dot{\phi}^{2}$. Because of the 3 -fold symmetry, one should consider the new angle variable $\theta_{R}=3 \phi$ since a rotation by $2 \pi / 3$ brings the rotor in the same state. Then, $E_{K}=\frac{1}{6} m_{p} a^{2} \dot{\theta}_{R}^{2}$. Consequently, $J_{R}=\frac{1}{6} m_{p} a^{2} \approx 1.0710^{-48}$. TET condition (15) or (43) becomes $\delta_{R}=0.5210^{-20}$ Joules $=3.210^{-2} \mathrm{eV}$. Since at quantum TET, we should have $2 n_{e}+1=4 D_{e} /\left(\hbar \omega_{e}\right)$, we obtain the dissociation energy $D_{e}=\left(n_{e}+1 / 2\right)^{2} \delta_{R} \approx n$ and $\hbar \omega_{e}=\left(2 n_{e}+1\right) \delta_{R}$ for a chemical bond conjugate to the $-C-H_{3}$ rotor where $n_{e}$ is the number of quantum vibrational states of this chemical bond. In order to fix the ideas, let us choose $n_{e}=5$, then $\hbar \omega_{e}=3.52 \cdot 10^{-1} \mathrm{eV}$ which is a rather large phonon frequency even beyond the maximum physical range. Then, $D_{e}=0.988 \mathrm{eV}$ which is a quite small dissociation energy especially for such a rigid bond. There are apparently no physical chemical bonds in this range of parameters.

However, a forthcoming paper demonstrates that TET may be also induced by Fermi resonance that is when the rotor frequency is a higher harmonics of the Morse oscillator frequency [41]. Then, chemical dissociation with the same model but different parameters remain almost as much as efficient even when resonance occurs by the fourth harmonics. We shall see that TET by Fermi resonance will turn to be more efficient for breaking chemical bonds with larger energy.

Actually, many kinds of heavier rotors involving atoms other than protons or even bigger molecular groups could be found in biomolecules. Those rotors in principle could break selectively the more energetic chemical bonds which are conjugate. It is also worthwhile to note that despite our model is classical, our selective chemical dissociation should be in principle sensitive to the isotopic substitution of the atoms involved since the frequencies of the normal modes would be changed which could break conjugacy. Otherwise, the origin of the small coupling is not important provided it is small. It could be due to a direct mechanical coupling by chemical groups or it could be electrostatic, dipolar, quadripolar ... or else.

We also briefly discussed in that paper, the effect of the quantum corrections, and concluded that this selective dissociation effect should manifest as a sharp peak in the quantum yield. Quantum corrections should not introduce major changes concerning the qualitative description of the classical process.

Coupling this two degree of freedom system with a complex environment is 
likely much more important. As we explained in section 2, one of the effect of the environment is to transform the anharmonic oscillators into local modes (at least for a coupling not too large). In other words, this local vibration is then "dressed" with a collective vibration of the environment which decays exponentially at long distance. The consequence is that Hamiltonians $H_{R}\left(I_{R}\right)$ and $H_{M}\left(I_{M}\right)$ are renormalized. Thus, the condition for TET should also renormalized and fulfilled for different parameters.

It should be also noted here that because of the fine tuning of TET, small change in the environment may be sufficient to detune or retune the two oscillators for conjugacy. This effect may be used to control the chemical dissociation thus opening or closing the chemical expressways with small external chemical or physical perturbations ( $\mathrm{pH}$, electric field, specific adsorbed molecules, etc...).

The second important consequence is due to the possible existence of many nonconjugate resonant modes in the environment. The simplest (but empirical) model which can be done for describing this complex situation, is to consider the environment as a harmonic phonon bath (with continuous spectrum) which results into extra damping terms in the dynamical equations. If the energy dissipation during TET is not too large (at the scale of the coupling energy), substantial energy transfer may still occur. We plan to correct the tuning conditions for TET in presence of damping and to find under which conditions, chemical dissociation may still be persist.

The third important consequence which is the most challenging is to understand the role the thermal fluctuations on the selective chemical dissociation. As already noted, the phonon bath could be modelled in the dynamical equation of the model as a random Langevin force. As the temperature increases, this random force is expected to gradually destroy the relative phase coherence of the rotor and Morse oscillator which is essential for TET. Then, energy transfer from the rotor to the Morse oscillator becomes gradually stochastic reducing the selectivity of the chemical dissociation. On contrary, if the oscillators are not well tuned one with each other, small thermal fluctuations may help at the beginning TET favoring chemical dissociation but only up to a certain certain temperature. Higher temperature would disfavor again the chemical dissociation. When the temperature increases too much and or when the oscillators are far from conjugacy, our model simply return to an genuine Kramers model which is essentially driven by a Brownian force and where the chemical expressways which could exist at low temperature have been washed out because of dynamical decoherence.

In any case, studying in details the effect of thermal fluctuations should reveal a variety of non trivial and interesting features interpolating low temperature coherent models with the standard Brownian theory of chemical reactions. 
Their investigation is left to further works.

In summary, although this simple model in this form will likely need improvements for applications, and despite we have no clearly identified example to propose in real systems, we believe that this model is conceptually very interesting. It explicits a simple mechanism which could produce a chemical expressway and thus show that this concept should not be considered as purely speculative. It is an intrinsically nonlinear phenomena which cannot exist in linear models. We hope that this result could open new directions for understanding biochemistry and especially concerning the high selectivity (and sensitivity) of many biochemical reactions while conventional approaches seem to fail.

\section{References}

[1] H. Kramers, Physica 7 (1940) 284-304

[2] P.Hänggi, P. Talkner and M. Borkovec, Reviews of Modern Physics 62 (1990) 251-341

[3] B.K. Burgess and D.J. Lowe, Chem.Phys. 96 (1996) 2983-3012

[4] S. Aubry and G. Kopidakis, IJMP B17 (22-24): 3908-3921 (2003); ibid. in Localization and Energy Transfer in Nonlinear Systems pp.1-27 (2003). World Scientific Publishing, Eds. L. Vásquez, R.S. MacKay and M.P. Zorzano; S. Aubry in Proceeding of Nonlinear Waves: Classical and Quantum Aspects (NATO ARW Estoril, 13 -17 July, 2003); S. Aubry and G. Kopidakis, to appear in J.Biol.Phys. (2005)

[5] http://tccc.iesl.forth.gr/cecam2004/cecam2004.html

[6] M. Joyeux, S.C. Farantos, R. Schinke, J. Phys.Chem. A106 (2002) 5407-5421

[7] R.T. Birge, H. Sponer, Phys.Rev. 28 (1926)259-283; J.W. Ellis, Phys.Rev. 33 (1929) 27-36

[8] Alwyn Scott,Nonlinear Science, Emergence and Dynamics of Coherent Structures, Oxford University Press (1999)

[9] A. J. Sievers and S. Takeno, Phys.Rev.Lett. 61 (1988) 97

[10] S. Aubry, Physica 103D (1997) 201-250

[11] S. Flach and C.R. Willis, Phys.Rep. 295 (1998) 181

[12] D.K. Campbell, S. Flach and Y.S. Kivshar, Physics Today 57 (2004) 43-59

[13] R.S. MacKay and S. Aubry, Nonlinearity 7 1623-1643 (1994)

[14] R.Livi, M. Spicci, R.S. MacKay, Nonlinearity 10 (1997) 1421-1434 
[15] S. Aubry, Ann. Inst.H.Poincarè, Phys. Théor. 68 (1998) 381-420

[16] S. Aubry, G. Kopidakis, V. Kadelburg, Discrete and Continuous Dynamical Systems, B1, (2001) 271-298

[17] G. James, J.Nonlinear Sci. 13 (2003) 27-63

[18] G. Kopidakis and S. Aubry, Physica D130 (1999) 155-186

[19] G. Kopidakis and S. Aubry, Physica D139 (2000) 247-275

[20] C. Albanèse and J. Fröhlich,Commun.Math.Phys. 138 (1991)193-205

[21] PROTEINS: Structure and Molecular Properties T.E. Creighton, W.H. Freeman and Cie (1997)

[22] S. Aubry and R. Schilling, in preparation

[23] A.S. Davydov, J.Theor.Biol. 38 (1973)559; ibid. Physica Satus Solidi B59 (1973) 465

[24] Davydov'Soliton Revisited: Self-Trapping of Vibrational Energy in Proteins eds. P.L. Christiansen and A.C. Scott NATO Science Series: B243 (1991)

[25] J. Edler, and P.Hamm, A.C. Scott Phys.Rev.Lett. 89067403

[26] J. Edler, P. Hamm Phys.Rev. B69 (2004) 214301

[27] J. Edler, R. Pfister, V. Pouthier, C. Falvo and P. Hamm Phys.Rev.Lett. 93 (2004) 106405

[28] M. Peyrard and A.R. Bishop,Phys.Rev.Lett. 62 (1989) 2755; M. Peyrard, Nonlinearity 17 (2004) R1-R40

[29] J. Farago and M. Peyrard, Physica D113 (1998) 297-306

[30] S. Aubry, G. Kopidakis, A. M. Morgante and G. P. Tsironis, Physica B296 (2001) 222

[31] G. Kopidakis, S. Aubry and G. P. Tsironis, Phys. Rev. Letts. 87, 165501 (2001)

[32] T. Eisenhammer, A. Hübler, T. Geisel, E. Lüscher, Phys.Rev. A41 (1990) 33323342

[33] C. Wargitsch and A.W. Hübler, Phys.Rev. E51 (1995) 1508-1519, L.E. Arsenault and A.W. Hubler, Phys.Rev. E51 (1995) 3561-3571

[34] D. Beigie and S. Wiggins, Phys.Rev. A45, 4803-4827 (1992)

[35] P.W. Atkin, Physical Chemistry Oxford University Press (1986)

[36] P. Maniadis, G. Kopidakis and S. Aubry, Physica 188D (2004) 153-177

[37] H. Taseli, J.Phys. A31 779-788 (1998)

[38] M.G. Benedict and B. Molnar, Phys.Rev. A60 R1737-1740 (1999) 
[39] R.J. Levis, G.M. Menkir, H. Rabitz, Science 292 ( April 27, 2001) 709-713

[40] Martin C. Gutzwiller, Chaos in Classical and Quantum Mechanics Springer (1990)

[41] P. Maniadis and S. Aubry, submitted to Physica D (2004) 